UNIVERSIDADE DE SÃO PAULO

FACULDADE DE ZOOTECNIA E ENGENHARIA DE ALIMENTOS DEPARTAMENTO DE MEDICINA VETERINÁRIA

ROBERTO ROMANO DO PRADO FILHO

INFLUÊNCIA DO DECÚBITO NA OCORRÊNCIA DE SHUNT PULMONAR EM OVINOS MANTIDOS EM ANESTESIA INALATÓRIA 
ROBERTO ROMANO DO PRADO FILHO

\section{INFLUÊNCIA DO DECÚBITO NA OCORRÊNCIA DE SHUNT PULMONAR EM OVINOS MANTIDOS EM ANESTESIA INALATÓRIA}

\section{Versão corrigida}

Dissertação apresentada à Faculdade de Zootecnia e Engenharia de Alimentos da Universidade de São Paulo, como requisito para a obtenção do Título de "Mestre em Ciências".

Área de Concentração: Biociência Animal

Orientador: Prof. Dr. Adriano Bonfim Carregaro

PIRASSUNUNGA 


\section{CERTIFICADO}

Certificamos que a proposta intitulada "Influência do decúbito na ocorrência de shunt pulmonar em ovinos mantidos em anestesia inalatória", protocolada sob o CEUA ne 1240030918 w0 001041), sob a responsabilidade de Adriano Bonfim Carregaro - que envolve a produção, manutenção e/ou utilização de animais pertencentes ao filo Chordata, subfilo Vertebrata (exceto o homem). para fins de pesquisa científica ou ensino - está de acordo com os preceitos da Lei 11.794 de 8 de outubro de 2008, com o Decreto 6.899 de 15 de julho de 2009, bem como com as normas editadas pelo Conselho Nacional de Controle da Experimentação Animal (CONCEA), e foi aprovada pela Comissăo de Ética no Uso de Animais da Faculdade de Zootecnia e Engenharia de Alimentos da Universidade de São Paulo - FZEA/USP (CEUA/FZEA) na reunião de 17/10/2018.

We certify that the proposal "Influence of recumbency upon the incidence of pulmonary shunt in inhalation-anaesthetized sheep ", utilizing 7 Ovines (males and females), protocol number CEUA 1240030918 (ID 001041), under the responsibility of Adriano Bonfim Carregaro - which involves the production, maintenance and/or use of animals belonging to the phylum Chordata, subphylum Vertebrata (except human beings), for scientific research purposes or teaching - is in accordance with Law 11.794 of October 8 , 2008, Decree 6899 of July 15, 2009, as well as with the rules issued by the National Council for Control of Animal Experimentation (CONCEA), and was approved by the Ethic Committee on Animal Use of the School of Animal Science and Food Engineering - (Säo Paulo University) (CEUA/FZEA) in the meeting of 10/17/2018.

Finalidade da Proposta: Pesquisa (Acadêmica)

Vigência da Proposta: de 10/2018 a 09/2019 Área: Medicina Veterinária

Origem: Prefeitura do Campus USP Fernando Costa

$\begin{array}{lllll}\text { Espécie: } & \text { Ovinos } & \text { sexo: } & \text { Machos e Fêmeas } & \text { idade: } 90 \text { a } 120 \text { dias } \quad \mathrm{N}: \quad 7 \\ \text { Linhagem: } & \text { Mestiço Dorper } & \text { Peso: } 35 \text { a } 40 \mathrm{~kg} & \end{array}$

Local do experimento; O experimento será realizado no Setor de Ruminantes da Unidade Didática Clínico-Hospitalar da Faculdade de Zootecnia e Engenharia de Alimentos da Universidade de Săo Paulo. Os animais serăo transportados para uma baia de internaçăo dois dias antes dos procedimentos. As baias terăo dimensöes de $3,5 \mathrm{~m} \times 4,0 \mathrm{~m} \times 6,0 \mathrm{~m}$ e cama de serragem de madeira. Uma vez na baia, será fornecido feno de capim tifton e água, ambos ad libitum. Para o procedimento, os animais serăo submetidos a jejum alimentar de 12 horas, sem restrição hidrica. Finalizados os procedimentos, os animais serão transportados de volta para o Setor de Ovinocultura da PUSP-FC.

Pirassununga, 01 de abril de 2021

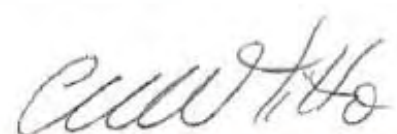

Profa. Dra. Cristiane Gonçalves Titto Coordenadora da Comissão de Ética no Uso de Animais Faculdade de Zootecnia e Engenharia de Alimentos da Universidade de Săo Paulo - FZEA/USP

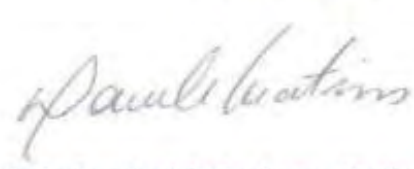

Profa. Dra. Daniele dos Santos Martins Vice-Coordenadora da Comissăo de Ética no Uso de Animais Faculdade de Zootecnia e Engenharia de Alimentos da Universidade de Săo Paulo - FZEAJUSP 
Ficha catalográfica elaborada pelo

Serviço de Biblioteca e Informação, FZEA/USP, com os dados fornecidos pelo(a) autor(a)

Prado Filho, Roberto Romano

Influência do decúbito na ocorrência de shunt pulmonar em ovinos mantidos em anestesia inalatória / Roberto Romano Prado Filho ; orientador Adriano Bonfim Carregaro. -- Pirassununga, 2021.

$50 \mathrm{f}$.

Dissertação (Mestrado - Programa de Pós-Graduação em Biociência Animal) - Faculdade de Zootecnia e Engenharia de Alimentos, Universidade de são Paulo.

1. Sevoflurano. 2. Shunt pulmonar. 3. Ovinos. 4. Ventilação mecânica. 5. Anestesia inalatória. I. Carregaro, Adriano Bonfim, orient. II. Título. 


\title{
INFLUÊNCIA DO DECÚBITO NA OCORRÊNCIA DE SHUNT PULMONAR EM OVINOS MANTIDOS EM ANESTESIA INALATÓRIA
}

\author{
Dissertação apresentada à Faculdade de \\ Zootecnia e Engenharia de Alimentos da \\ Universidade de São Paulo, como parte dos \\ requisitos para a obtenção do Título de Mestre \\ em Ciências.
}

Área de Concentração: Biociência Animal

Data de aprovação:

Banca Examinadora:

Prof. Dr.

Instituição

Presidente da Banca Examinadora

Prof.(a) Dr.(a) Instituição

Prof.(a) Dr.(a) Instituição

Prof.(a) Dr.(a) Instituição 
Miminha Belissima Cesposa. Matale Pravia Martins Romano, minha inspirasãa e exemplo de vida, minha fortaleza e meu porto seguro, dedico esta dissertasãa. 


\section{AGRADECIMENTOS}

Ao Nosso Grande Deus e Senhor, Jesus Cristo, cuja misericórdia e amizade pela humanidade tornaram possível a execução deste estudo. À Santíssima Mãe de Deus e a meu Anjo da Guarda, pelas orações constantes no decorrer deste mestrado.

À minha amada esposa, Natalí Faria Martins Romano, por todo amor e apoio incondicionais que me deu no período do mestrado. Por todos os momentos, bons e ruins que passamos juntos na pós-graduação. Por me fazer perceber, a todo instante, que seremos sempre um pelo outro. Você é o melhor presente que eu poderia ganhar em toda a minha vida. À minha querida filha, Ana Clara Martins Romano, que nos trouxe uma felicidade incomparável, e a certeza de que podemos contar com a Divina Providência.

A meus pais, Analice e Roberto, por terem acreditado em mim a todo instante, desde o vestibular até o fim da pós-graduação, me educando com valores sólidos e perpétuos, dos quais jamais esquecerei. Vocês sempre serão o meu exemplo de vida matrimonial, honestidade, caráter e superação das adversidades. Que o Senhor Nosso Deus lhes conceda longos dias na Terra que Ele fez para nós.

Ao Prof. Dr. Adriano Bonfim Carregaro, por toda a orientação e paciência que recebi durante o mestrado, desde a confecção do projeto inicial, que precisou ser totalmente reformulado, passando pelo experimento em meio à pandemia e ao final com o artigo e a dissertação. Apesar da minha imaturidade intelectual e dos inúmeros conflitos que tivemos ao longo da minha trajetória na pós-graduação, prevaleceu o exemplo de um docente que será sempre um modelo de profissionalismo, disciplina, cuidado com os pacientes, excelência na anestesia, retidão de intenção, vontade de fazer sempre o correto, dentre muitas outras virtudes, as quais levarei comigo por toda a vida. Agradeço não apenas por ter me orientado na pósgraduação, como também me aconselhado, sempre que possível, na graduação e na residência.

À Profa. Dra. Thais Feres Bressan, por todo o auxílio durante a ausência do Prof. Adriano, tanto na rotina quanto no exame de qualificação. Agradeço também aos meus colegas do Núcleo de Anestesiologia Veterinária (NAVE): Jeff, Nina, Julia, Thais Macedo e Nathalia. À Mariana Sanches, pela ajuda com as anestesias das ovelhas em meio à pandemia. Foi uma honra conviver com todos, ainda que por pouco tempo.

Aos amigos do CEPTOX/FMVZ, Estevão Belloni e Marco Antônio Ferreira dos Santos, pela ajuda diária com o manejo e cuidado das ovelhas. Aprendi muito, diariamente, com vocês, muito além de criação animal, e lamento que não tenhamos convivido por mais tempo. Ao amigo Rildo Alexandre, pela parceria e pela ajuda no cuidado das ovelhas enquanto estavam na UDCH para serem anestesiadas. 
Ao Prof. Dr. Eduardo Harry Birgel Junior, por ter gentilmente cedido uma baia de internação e um espaço no centro cirúrgico do setor de Ruminantes da UDCH para que o projeto pudesse ser executado.

Às ovelhas Albânia, Armênia, Bulgária, Capadócia, Georgia, Jordânia, Pisidia, Romênia, Sérvia e Turquia, que literalmente deram as suas vidas para que esse trabalho pudesse ser executado.

À Faculdade de Zootecnia e Engenharia de Alimentos da Universidade de São Paulo e ao Programa de Pós-Graduação em Biociência Animal, pela oportunidade do mestrado.

À Fundação de Amparo à Pesquisa do Estado de São Paulo (FAPESP), pelo financiamento do processo no 2018/15165-9, e à Coordenação de Aperfeiçoamento de Pessoal de Nível Superior (CAPES).

A todos que de alguma forma contribuíram para que este trabalho pudesse ser realizado, o meu eterno agradecimento. 
"Ofruto da virtude é a perseveransa. Ofruto da perseveransa é a hábito, e ofruto do hábito é o caráter."

São João Clímaco (séc. VI d.C.) 


\section{RESUMO}

PRADO FILHO, R. R. Influência do decúbito na ocorrência de shunt pulmonar em ovinos mantidos em anestesia inalatória. 2021. 50 f. Dissertação (Mestrado). - Faculdade de Zootecnia e Engenharia de Alimentos, Universidade de São Paulo, Pirassununga, 2021.

O presente estudo teve por objetivo determinar o impacto de diferentes decúbitos durante a anestesia inalatória de ovinos, priorizando o estudo das alterações ventilatórias e de shunt pulmonar. Para esse fim, sete ovelhas foram submetidas a anestesia inalatória com sevofluorano e mantidas em ventilação mecânica controlada por pressão $\left(12 \mathrm{cmH}_{2} \mathrm{O}\right)$, com $f$ fixada em 10 mpm e pressão positiva ao final da expiração em $0 \mathrm{~cm} \mathrm{H}_{2} \mathrm{O}$ (ZEEP). Imediatamente após indução anestésica, os animais foram submetidos a três diferentes tratamentos, a saber: decúbitos dorsal, lateral esquerdo ou lateral direito. A ordem dos tratamentos foi determinada de forma aleatória, sendo que todos os animais foram submetidos a todos os tratamentos, com duração de 120 minutos cada. O intervalo entre os procedimentos foi de 48 horas. Todos os parâmetros foram adaptados do projeto inicial após estudo-piloto, o mesmo ocorrendo com a adequação do intervalo, o qual não influenciou nos resultados do estudo-piloto. Foram coletadas amostras de sangue arterial e venoso para análises de gases sanguíneos e eletrólitos, imediatamente após indução anestésica, decorridos 60 minutos da indução e após 120 minutos da indução. Os valores obtidos foram utilizados para calcular a fração de shunt pulmonar (V/Q), conteúdo arterial de oxigênio $\left(\mathrm{CaO}_{2}\right)$, conteúdo venoso central de oxigênio $\left(\mathrm{CcvO}_{2}\right)$, conteúdo capilar de oxigênio $\left(\mathrm{Cc}^{\prime} \mathrm{O}_{2}\right)$, pressão alveolar de oxigênio $\left(\mathrm{PAO}_{2}\right)$, volume minuto $(\mathrm{VM})$, volume corrente ( $\mathrm{VCe})$, gradiente alvéolo-arterial de oxigênio $\left[\mathrm{P}(\mathrm{A}-\mathrm{a}) \mathrm{O}_{2}\right]$ e gradiente de dióxido de carbono expirado e arterial [(a-ET) $\mathrm{CO}_{2}$ ]. Os resultados mostraram que V/Q diminuiu expressivamente de 0 a 120 minutos em todos os grupos (dorsal: 69,3\% para 27,3\%; lateral esquerdo: 59,1\% para 25,0\%; lateral direito: $67,2 \%$ para $32,4 \%$ ). Os valores de $\mathrm{CaO}_{2}, \mathrm{CcvO}_{2}$, $\mathrm{CcO}_{2}, \mathrm{PAO}_{2}$, e $\mathrm{P}(\mathrm{A}-\mathrm{a}) \mathrm{O}_{2}$ foram significativamente mais elevados em todos os grupos, a partir de 60 minutos após a indução, e mantendo-se posteriormente $(\mathrm{p}<0.05)$. Níveis mais elevados de $\mathrm{CaO}_{2}$ foram observados nos animais do grupo lateral esquerdo, no minuto $0(9,9 \pm 1,43$ $\left.\mathrm{mL} \cdot \mathrm{dL}^{-1}\right)$ em comparação com o decúbito dorsal $\left(8,9 \pm 1,4 \mathrm{~mL} \cdot \mathrm{dL}^{-1}\right)(\mathrm{p}=0.0177)$. Não foram observadas diferenças entre tratamentos para $\mathrm{CcvO}_{2}, \mathrm{CcO}_{2}$ e $\mathrm{PAO}_{2}$. O grupo lateral esquerdo apresentou menores valores de $\mathrm{P}(\mathrm{A}-\mathrm{a}) \mathrm{O}_{2}$ em 0 min $(\mathrm{p}=0,0117)$ e 60 min em relação ao direito $(p=0,0282)$. Não foram encontradas diferenças nem VT e VM em todos os tratamentos e entre os momentos, e o mesmo foi observado para os valores de $\mathrm{P}(\mathrm{a}-\mathrm{ET}) \mathrm{CO}_{2}$. A $\mathrm{PaO}_{2}$ apresentou valores mais elevados aos 60 e 120 minutos, em todos os grupos, quando comparada ao minuto 0 ( $\mathrm{p}<0,001$ ). Por outro lado, o grupo dorsal apresentou $\mathrm{PaCO}_{2}$ estável durante todo o período de anestesia, sendo diferente dos demais aos 120 minutos. Conclui-se que o decúbito exerce pouca influência na formação de shunt pulmonar.

Palavras-chave: sevoflurano; ovelhas; shunt pulmonar; ventilação mecânica; 


\begin{abstract}
PRADO FILHO, R. R. Influence of recumbency upon pulmonary shunt occurrence in inhalation-anaesthetized sheep. 2021. 50 f. M.Sc. Dissertation - Faculdade de Zootecnia e Engenharia de Alimentos, University of Sao Paulo, Pirassununga, 2021.

The present study analyzed the impact of different decubitus in sheep during inhalation anaesthesia, prioritizing ventilatory changes and pulmonary shunt. For this purpose, 7 female sheep were submitted to sevoflurane inhalation anesthesia, maintained on pressure-controlled ventilation $\left(12 \mathrm{cmH}_{2} \mathrm{O}\right)$, respiratory rate $(f)$ fixed on $10 \mathrm{mpm}$ and a zero end-expiratory pressure (ZEEP). Immediately after anesthetic induction, the animals undergone three different treatments, namely: dorsal, left lateral or right lateral recumbency positions. Treatments order were determined randomly, being all animals subjected to all treatments, with duration of 120 minutes each. Interval between procedures was 48 hours. Arterial and central venous blood samples were collected for blood gas and electrolytes analysis immediately after anesthetic induction, after 60 minutes of induction and after 120 minutes of induction. Obtained values were used to calculate the pulmonary shunt fraction $(\mathrm{V} / \mathrm{Q})$, arterial oxygen content $\left(\mathrm{CaO}_{2}\right)$, central venous oxygen content $\left(\mathrm{CcvO}_{2}\right)$, pulmonary end-capillary oxygen content $\left(\mathrm{Cc}^{\text {' }} \mathrm{O}_{2}\right)$, alveolar oxygen partial pressure $\left(\mathrm{PAO}_{2}\right)$, minute volume $\left(\mathrm{V}_{\mathrm{M}}\right)$, tidal volume $\left(\mathrm{V}_{\mathrm{T}}\right)$, alveolararterial oxygen gradient $\left[\mathrm{P}(\mathrm{A}-\mathrm{a}) \mathrm{O}_{2}\right]$ and arterial-end-tidal carbon dioxide gradient $\left[(\mathrm{a}-\mathrm{ET}) \mathrm{CO}_{2}\right]$. Results showed that V/Q greatly decreased from 0 to $120 \mathrm{~min}$ in all the groups (dorsal: $69.3 \%$ to $27.3 \%$; left lateral: $59.1 \%$ to $25.0 \%$; right lateral: $67.2 \%$ to $32.4 \%$ ). $\mathrm{CaO}_{2}, \mathrm{CcvO}_{2}, \mathrm{CcO}_{2}$, $\mathrm{PAO}_{2}, \mathrm{P}(\mathrm{A}-\mathrm{a}) \mathrm{O}_{2}$ values were significantly higher in all the groups, from $60 \mathrm{~min}$ after induction, and maintaining them further $(\mathrm{p}<0.05)$. Higher $\mathrm{CaO}_{2}$ levels in animals from the left lateral recumbency group were observed at $0 \mathrm{~min}\left(9.9 \pm 1.43 \mathrm{~mL} \cdot \mathrm{dL}^{-1}\right)$ compared to the dorsal recumbency group $\left(8.9 \pm 1.4 \mathrm{~mL} \cdot \mathrm{dL}^{-1}\right)(\mathrm{p}=0.0177)$. No differences between treatments were observed for $\mathrm{CcvO}_{2}, \mathrm{CcO}_{2}$ and $\mathrm{PAO}_{2}$. The left recumbency group showed lower $\mathrm{P}(\mathrm{A}-\mathrm{a}) \mathrm{O}_{2}$ values at $0 \mathrm{~min}(\mathrm{p}=0.0117)$ and $60 \mathrm{~min}$ compared to the right one $(\mathrm{p}=0.0282)$. No differences were found in $\mathrm{V}_{\mathrm{T}}$ and $\mathrm{V}_{\mathrm{M}}$ in all treatments and between time points, and the same was observed for $\mathrm{P}(\mathrm{a}-\mathrm{ET}) \mathrm{CO}_{2}$ values. $\mathrm{FiO}_{2}$, $\mathrm{HR}$ and $\mathrm{FE}$ 'Sevo showed no statistical difference at different time points. $\mathrm{PaO}_{2}$ showed higher values at 60 and $120 \mathrm{~min}$ for all the groups, when compared to the 0 minute $(\mathrm{p}<0.001) . \mathrm{PaCO}_{2}$ followed the same pattern, but only for the right and left recumbency groups. Conversely, the dorsal recumbency group showed stable $\mathrm{PaCO}_{2}$ throughout the anaesthesia period, thus, being different from the others at $120 \mathrm{~min}$. In conclusion, recumbency has little influence on pulmonary shunt occurrence.
\end{abstract}

Keywords: sevoflurane, sheep, pulmonary shunt, mechanical ventilation. 


\section{LISTA DE FIGURAS}

Figure 1 - Mean \pm ED of the pulmonary shunt fraction (V/Q) of sevoflurane-anaesthetized sheep, mechanically-ventilated under dorsal, right lateral or left lateral recumbencies. * Statistical difference from 0 minute; Different letters indicate statistical difference between groups $(\mathrm{P} \leq 0.05)$

\section{LISTA DE TABELAS}

Table 1 - Measured oxygen tension, content indexes and parameters of sevofluraneanaesthetized sheep, mechanically-ventilated under dorsal, right lateral or left lateral recumbencies.

Table 2 - Measured physiological and blood gas parameters of sevoflurane-anaesthetized sheep, mechanically-ventilated under dorsal, right lateral or left lateral recumbencies. 


\section{SUMÁRIO}

1 INTRODUÇÃOO 14

2 OBJETIVOS 16

2.1 OBJETIVOS GERAIS 16

2.2 OBJETIVOS ESPECÍFICOS 16

3 HIPÓTESES 16

4 CAPÍTULO 1 - REVISÃO DE LITERATURA - SHUNT PULMONAR 17

REFERÊNCIAS 26

5 CAPÍTULO 2 - INFLUENCE OF RECUMBENCY ON THE PULMONARY SHUNT

IN SEVOFLURANE-ANAESTHETIZED SHEEP 32

$\begin{array}{ll}\text { ABSTRACT } & 32\end{array}$

$\begin{array}{ll}\text { RESUMO } & 33\end{array}$

5.1 INTRODUCTION 34

5.2 MATERIALS AND METHODS 35

$\begin{array}{ll}5.3 \text { RESULTS } & 37\end{array}$

5.4 DISCUSSION 38

$\begin{array}{ll}5.5 \text { CONCLUSION } & 40\end{array}$

ACKNOWLEDGEMENTS $\quad 41$

REFERENCES 41

$\begin{array}{ll}\text { APENNDICE A } & 50\end{array}$ 


\section{INTRODUÇÃO}

Bovinos e ovinos estão sujeitos à hipoventilação e hipoxemia quando são posicionados em decúbito dorsal (KLEIN; FISHER, 1988). Os órgãos da cavidade abdominal destes animais exercem pressão sobre o diafragma, resultando em compressão pulmonar, redução da capacidade de ventilação espontânea e do fluxo sanguíneo pulmonar, com formação de shunt pulmonar (KLEIN; FISHER, 1988; MEYER et al., 2010; WAGNER; MUIR; GROSPITCH, 1990).

Os ovinos são amplamente utilizados em pesquisas biomédicas odontológicas e ortopédicas (SARTORETTO et al., 2016), que envolvem procedimentos cirúrgicos prolongados nos quais o plano anestésico é melhor alcançado em anestesia geral inalatória (GRAY; MCDONELL, 1986). Apesar do custos, procedimentos anestésicos são necessários em ovinos para facilitar diagnósticos ou cirurgias, especialmente em animais reprodutores, de alto valor zootécnico, e vezes também em animais de companhia (WHITE; TAYLOR, 2000).

A condição de shunt pulmonar ocorre quando não há correta oxigenação dos alvéolos, embora encontrem-se corretamente perfundidos (LOVERING et al., 2015). Tal condição está diretamente relacionada aos distúrbios na relação ventilação/perfusão pulmonar (WAGNER; MUIR; GROSPITCH, 1990). Estratégias na redução destas condições incluem períodos de jejum alimentar pré-anestésicos (BLAZE; LEBLANC; ROBINSON, 1988) e algumas variações de decúbito dorsal (PERILLI et al., 2003). Considera-se uma pequena porcentagem (10\%) de shunt como sendo fisiológica (LUMB, 2000). Durante anestesia, este valor pode atingir até 30\%, sem suplementação de oxigênio (ARAOS et al., 2012).

A redução do volume pulmonar é a principal causa de shunt em ovelhas submetidas à anestesia inalatória (DUECK; RATHBUN; GREENBURG, 1984), e que pode ser agravada dependendo do decúbito (WHITE; TAYLOR, 2000). A compressão diafragmática que ocorre devido à distensão ruminal, por redução da atividade do órgão ou atonia, reduz substancialmente a complacência pulmonar, principalmente quando estes animais são posicionados em decúbito lateral ou dorsal (MEYER et al., 2010). O decúbito lateral está associado à potencialização dos efeitos deletérios da compressão diafragmática na ventilação de ovelhas (FUJIMOTO; LENEHAN, 1985). Em decúbito dorsal, com posição de Trendelenburg reversa, não se observou piora da função cardiovascular ou melhora da função respiratória de bovinos (ARAÚJO et al., 2017). Por outro lado, sugere-se que há melhor estabilidade ventilatória e oxigenação tecidual em ovelhas submetidas a ventilação mecânica e posicionadas em decúbito dorsal (ROSA, 2017). 
A importância de se estudar diferentes decúbitos decorre dos diferentes impactos na anestesia de ovinos, potencializando possíveis incompatibilidades na relação ventilaçãoperfusão. Em virtude da invasibilidade dos procedimentos cirúrgicos a que estes animais são submetidos, e sendo a anestesia geral inalatória fundamental para que os ocorram de forma ética e com menor risco possível, torna-se imprescindível que se determine a influência dos diferentes tipos de decúbito sobre os parâmetros cardiopulmonares a fim de se evitar shunt pulmonar no período transanestésico. 


\section{OBJETIVOS}

\subsection{OBJETIVOS GERAIS}

- Determinar o impacto de diferentes decúbitos durante a anestesia inalatória de ovinos, priorizando o estudo das alterações ventilatórias e de shunt pulmonar.

- Comparar os efeitos cardiopulmonares dos decúbitos lateral esquerdo, direito e dorsal em ovinos durante anestesia inalatória com sevoflurano, mantidos em ventilação artificial.

\subsection{OBJETIVOS ESPECÍFICOS}

- Avaliar a eficiência das trocas gasosas por meio das variáveis pressão arterial de dióxido de carbono $\left(\mathrm{PaCO}_{2}\right)$, pressão arterial de oxigênio $\left(\mathrm{PaO}_{2}\right)$, pressão venosa central de dióxido de carbono $\left(\mathrm{PcvCO}_{2}\right)$ e pressão venosa central de oxigênio $\left(\mathrm{PcvO}_{2}\right)$.

- Determinar a fração de shunt pulmonar utilizando a fração de oxigênio inspirado $\left(\mathrm{FiO}_{2}\right)$, e valores de índices de tensão de oxigênio, a saber, conteúdo de oxigênio arterial $\left(\mathrm{CaO}_{2}\right)$, conteúdo venoso central de oxigênio $\left(\mathrm{CcvO}_{2}\right)$ e conteúdo de oxigênio capilar $\left(\mathrm{Cc}^{\prime} \mathrm{O}_{2}\right)$.

\section{HIPÓTESES}

- O posicionamento em diferentes decúbitos determina a magnitude do shunt pulmonar nos ovinos. 


\section{CAPÍTULO 1 - REVISÃO DE LITERATURA - SHUNT PULMONAR}

Modelos experimentais animais são amplamente utilizados na pesquisa científica para aperfeiçoar e validar procedimentos existentes, desenvolver novos materiais e compreender processos fisiológicos e patológicos, uma vez que modelos in vitro não são capazes de reproduzir completamente a complexidade da fisiologia humana (CALASANS-MAIA et al., 2009). Os ovinos, quando utilizados como modelo experimental, permitem o desenvolvimento de conhecimento básico por meio de estudos pré-clínicos e clínicos, visando melhorias na saúde e bem-estar humano e animal (MARTINI et al., 2001; SARTORETTO et al., 2016).

Os diferentes modelos animais para experimentação podem ser classificados em pequenos animais (camundongos, ratos e cobaias), médios animais (coelhos, gatos, miniporcos e cães) e grandes animais (equinos, ovinos e caprinos). Quando da escolha de uma espécie animal, deve-se considerar os seguintes critérios, a saber: 1) princípios éticos; 2) facilidade e adaptabilidade à manipulação experimental; 3) custo e disponibilidade; 4) possibilidade de estudo de fenômenos biológicos ou comportamento animal; 5) investigação de resposta tecidual induzida ou espontânea; 6) uniformidade genética entre os animais, quando aplicável; 7) sobrepujar dados para outros animais ou humanos; 8) resistência à doenças ou infecções; 9) tempo de vida dos animais em relação à duração dos estudos; e 10) capacidade de desenvolver experimentos que mimetizem situações clínicas em outras espécies (FAGUNDES; TAHA, 2004; PEARCE et al., 2007).

Dentre os modelos experimentais, a espécie ovina é uma das mais utilizadas, dentre outras características, por mimetizar condições clínicas semelhantes à de humanos (TURNER, 2007). Os ovinos são frequentemente utilizados como modelos de estudo na biomedicina com objetivo de testar biomateriais para uso humano (SIDDIQI et al., 2016). Destaca-se o uso de ovinos em experimentos envolvendo modelos de implantes dentários, mandibulares e maxilares (BARBONI et al., 2013; FRISKEN et al., 2002; LE et al., 2018; TATARA et al., 2016), bem como de cirurgias ortopédicas (CLAES et al., 1998; LERNER et al., 2015; WALSH et al., 2007), uma vez que o tempo de cicatrização óssea nesta espécie é muito semelhante à espécie humana (EGERMANN; GOLDHAHN; SCHNEIDER, 2005). Ademais, há contribuição notória dos ovinos na compreensão de processos fisiopatológicos de interesse na medicina humana, evidenciando-se: pneumologia, na qual os ovinos servem como modelo de estudo para doenças respiratórias agudas; neonatologia, na qual patologias associadas a fetos humanos são reproduzidas e estudadas em fetos ovinos; obstetrícia, onde a ovelha gestante serve como modelo para gestação humana; e cardiologia, na qual modelos de implantes cardíacos são 
primeiramente estudados em ovinos. (BARRY; ANTHONY, 2008; FLANAGAN et al., 2009; FUJINO et al., 2001; HILLMAN et al., 2007).

Durante a experimentação realizada com modelos animais para fins educacionais ou de pesquisa, é de fundamental importância minimizar desconforto, estresse e dor ao longo dos procedimentos (CARBONE, 2011). Tais procedimentos, que podem causar dor momentânea, devem ser minimamente realizados ou evitados ao máximo, ou ainda, em casos extremos de necessidade científica, sob sedação, analgesia ou anestesia (WATANABE; FONSECA; VATTIMO, 2014). Desta maneira, recomenda-se o uso de anestesia geral inalatória nestes animais quanto maior for a invasibilidade dos procedimentos (MEEUSEN et al., 2009; MURAKAMI et al., 2002).

A anestesia inalatória torna possível controlar o plano anestésico e a duração da anestesia sem exceder o tempo de recuperação anestésica (MOHAMADNIA; HUGHES; CLARKE, 2008), e requer mínima biotransformação dos agentes inalatórios, visto que a grande maioria é eliminada de forma inerte por via pulmonar, e promove menor efeito cumulativo mesmo após longos períodos de anestesia (ELLIOTT; STRUNIN, 1993). Além disto, a anestesia inalatória auxilia na redução de morbidade e mortalidade dos pacientes, porquanto facilita a ventilação pulmonar e melhora a oxigenação arterial. Ainda, os agentes inalatórios, quando em alíquotas, podem ser continuamente mensurados, fato que aumenta a precisão e a segurança desta modalidade anestésica em relação à anestesia total intravenosa (STEFFEY; MAMA; BROSNAN, 2015).

Os principais fármacos utilizados em anestesias inalatórias na medicina veterinária são isofluorano, sevofluorano e desfluorano (STEFFEY; MAMA; BROSNAN, 2015). Os efeitos cardiopulmonares do desfluorano e sevofluorano são bem descritos em felinos, sendo semelhantes aos do isofluorano, com exceção de que a frequência cardíaca tende a ser inferior com sevofluorano (HIKASA et al., 1996). Os três agentes causam diminuição dose-dependente na pressão arterial, resistência vascular sistêmica e, em doses elevadas, depressão miocárdica e diminuição do débito cardíaco em cães e gatos (CLARKE, 1999; MUTOH et al., 1997).

Entretanto, algumas particularidades na anestesia inalatória dos ovinos devem ser consideradas, dado que pequenos ruminantes secretam grande quantidade de saliva (GALATOS, 2011), e que o jejum inadequado pode levar ao regurgitamento de conteúdo ruminal (CARROLL; HARTSFIELD, 1996), resultando em obstrução e aspiração das vias aéreas superiores com consequências potencialmente fatais, incluindo asfixia e pneumonias no período pós-operatório (TRANQUILLI, 1986). Os riscos de obstrução e aspiração continuam durante o período de recuperação anestésica, até que o animal tenha recuperado o controle de 
seus reflexos faríngeo e laríngeo e possa se manter em decúbito esternal (HALL; CLARKE; TRIM, 2001).

O rúmen ovino ocupa cerca de três quartos da cavidade abdominal e sua proximidade com o diafragma pode interferir na ventilação durante o ato anestésico (EWING, 1990). A ventilação espontânea em animais posicionados em decúbito dorsal ou lateral se torna comprometida devido ao peso ruminal, bem como do útero gravídico quando presente, fato que pode desencadear hipoventilação, hipóxia e hipercapnia (BLAZE; LEBLANC; ROBINSON, 1988). Além disso, os decúbitos laterais ou dorsal, sedação intensa e anestesia geral prejudicam a eructação; o gás produzido pela fermentação contínua da ingesta se acumula no rúmen podendo levar ao timpanismo, o que pode agravar ainda mais o desconforto respiratório (VALVERDE; DOHERTY, 2008). Quando o animal permanece em decúbito dorsal, as vísceras abdominais, incluindo o rúmen, comprimem os principais vasos abdominais e impedem o retorno do sangue venoso. Por conseguinte, há diminuição do débito cardíaco, da pressão arterial e da perfusão tecidual (GALATOS, 2011). Recomenda-se jejum alimentar de 12 a 18 horas e jejum hídrico de 6 horas para que os efeitos supramencionados sejam minimizados (WHITE; TAYLOR, 2000).

O comprometimento na ventilação durante anestesia inalatória pode levar à incompatibilidade na relação ventilação/perfusão pulmonar (STEFFEY, 1986). A anestesia geral inalatória está associada à redução da capacidade residual funcional (CRF) e deficiências nas trocas gasosas (HICKEY et al., 1973), suscitando em distribuição alterada dos gases inspirados, redução de volume pulmonar, obstrução de vias aéreas e hipóxia pulmonar (REHDER et al., 1971). A obstrução das vias aéreas por compressão pulmonar, bem como a perda de surfactante, e a reabsorção dos gases alveolares acarretam em atelectasia dos pulmões (DUGGAN; KAVANAGH, 2005).

A compressão pulmonar ocorre quando a pressão de distensão do alvéolo se reduz a um nível que permite que o mesmo colapse. Após indução anestésica, o diafragma relaxa e se desloca cranialmente, pelo peso das vísceras abdominais, comprimindo as regiões pulmonares adjacentes (BRISMAR et al., 1985). Outra causa de atelectasia pulmonar, a reabsorção dos gases alveolares pode ocorrer de duas formas. Primeiro, após a completa oclusão das vias aéreas, uma bolsa de gás retido é criada na unidade pulmonar distal à obstrução. Dessa forma, a absorção de gás pelo sangue continua e a entrada de gás é impedida pelas vias aéreas bloqueadas, fazendo com que a bolsa de gás entre em colapso (LORING; BUTLER, 1987). 
Em segundo lugar, regiões com baixa ventilação em comparação com a perfusão terão baixas tensões de oxigênio alveolar quando a fração inspirada de oxigênio $\left(\mathrm{FiO}_{2}\right)$ estiver baixa. Quando a $\mathrm{FiO}_{2}$ é aumentada (ou seja, com oxigênio suplementar), a tensão de oxigénio alveolar $\left(\mathrm{PAO}_{2}\right)$ aumenta, e a velocidade da transferência de gás alveolar para o sangue capilar também aumenta. Além disso, à medida que o $\mathrm{PAO}_{2}$ aumenta, a tensão do nitrogênio alveolar $\left(\mathrm{PAN}_{2}\right)$ diminui. Como o nitrogênio é insolúvel, qualquer absorção aumentada de oxigênio resultará na perda do volume alveolar e em colapso (REHDER et al., 1979; WAGNER et al., 1974).

Por consequência, o processo de atelectasia pulmonar e as trocas gasosas prejudicadas geram o shunt pulmonar (ROTHEN et al., 1996). Define-se como shunt pulmonar a condição patológica na qual há baixa relação ventilação/perfusão pulmonar, isto é, os alvéolos estão normalmente perfundidos, porém, com debilidade nas trocas gasosas (GARAY; KAMELAR, 1989). Essa condição pode ser agravada em situações de decúbito, nas quais especialmente o volume ruminal pressiona continuamente o diafragma (BLAZE; LEBLANC; ROBINSON, 1988). A redução do volume pulmonar é a principal causa de desenvolvimento de shunt pulmonar em ovinos submetidos a anestesia inalatória (DUECK; RATHBUN; GREENBURG, 1984).

O shunt pulmonar pode ser calculado por meio de índices de tensão e conteúdo de oxigênio específicos. (PETERSSON; GLENNY, 2014). O valor da fração do shunt pulmonar pode ser obtido por meio de métodos invasivos ou minimamente invasivos (REES et al., 2003). A equação para calcular o shunt pulmonar é usada como base sobre a qual os índices de oxigenação arterial e troca gasosa podem ser estudados, podendo ser expresso pela seguinte fórmula (WEISKOPF; SEVERINGHAUS, 1972):

$$
V / Q=\left(\mathrm{Cc}^{\prime} \mathrm{O}_{2}-\mathrm{CaO}_{2}\right) /\left(\mathrm{Cc}^{\prime} \mathrm{O}_{2}-\mathrm{CvO}_{2}\right)
$$

No qual $V / Q$ é a fração de shunt pulmonar, $C c^{\prime} O_{2}$ é o conteúdo capilar de oxigênio, $\mathrm{CaO}_{2}$ é o conteúdo arterial de oxigênio e $\mathrm{CvO}_{2}$ é o conteúdo de oxigênio da mistura venosa Considera-se o valor de $10 \%$ como sendo shunt anatômico ou fisiológico (LUMB, 2000). Em ovinos anestesiados, este valor pode atingir até aproximadamente $30 \%$, sem suplementação de oxigênio (ARAOS et al., 2012).

O conteúdo arterial de oxigênio sanguíneo depende de vários fatores, incluindo a pressão parcial do oxigênio inspirado, a adequação da ventilação e das trocas gasosas, a concentração de hemoglobina e a afinidade da molécula de hemoglobina pelo oxigênio. Do oxigênio transportado pelo sangue, uma proporção muito pequena é dissolvida em solução simples, com a grande maioria quimicamente ligada à molécula de hemoglobina nas hemácias, 
sendo tal processo passível de reversibilidade (O'DRISCOLL et al., 2008). Dessa maneira, o conteúdo arterial de oxigênio de pode ser calculado pela seguinte fórmula (STAUB, 1963):

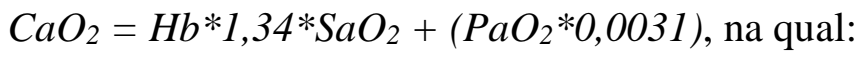

$H b$ é a concentração sérica de hemoglobina ( $\mathrm{g} / \mathrm{dL})$; 1,34 é o volume de oxigênio carreado pela hemoglobina totalmente saturada; $\mathrm{SaO}_{2}$ é a fração de hemoglobina arterial saturada com oxigênio; $\mathrm{PaO}_{2}$ é a pressão parcial de oxigênio arterial; 0,0031 é o coeficiente de solubilidade de Bunsen para oxigênio plasmático.

$\mathrm{O} \mathrm{CaO}_{2}$ é expresso em $\mathrm{mL}$ de oxigênio por $100 \mathrm{~mL}$ ou por $\mathrm{L}$ de sangue, enquanto a saturação de oxigênio arterial $\left(\mathrm{SaO}_{2}\right)$ é expressa como uma porcentagem que representa o total geral de locais de ligação de oxigênio na hemoglobina. Em indivíduos saudáveis que respiram ar ambiente, ao nível do mar, $\mathrm{SaO}_{2}$ apresenta valores entre $96 \%$ e $98 \%$. O volume máximo de oxigênio que o sangue pode transportar quando totalmente saturado é denominado capacidade de transporte de oxigênio, que, em uma concentração normal de hemoglobina, é de aproximadamente $20 \mathrm{~mL}$ de oxigênio por $100 \mathrm{~mL}$ de sangue (COLLINS et al., 2015).

O conteúdo de oxigênio da mistura venosa depende também dos determinantes da capacidade de transporte de oxigênio, tais como pressão parcial venosa mista de oxigênio, saturação de oxigênio venosa mista, concentração de hemoglobina e a afinidade da molécula de hemoglobina pelo oxigênio (PEARSE; RHODES, 1970). Além disso, o $\mathrm{CvO}_{2}$ depende da quantidade de oxigênio anterior à formação de sangue venoso, da quantidade de sangue arterial transportada para os tecidos e, de quanto oxigênio foi extraído do sangue arterial para os tecidos (REINHART, 2004). O conteúdo de oxigênio da mistura venosa pode ser calculado pela seguinte fórmula (STAUB, 1963):

$$
\mathrm{CvO}_{2}=\mathrm{Hb}^{*} 1,34 * \mathrm{SvO}_{2}+\left(\mathrm{PaO}_{2} * 0,0031\right) \text {, na qual: }
$$

$H b$ é a concentração sérica de hemoglobina $(\mathrm{g} / \mathrm{dL}) ; 1,34$ é o volume de oxigênio carreado pela hemoglobina totalmente saturada; $\mathrm{SvO}_{2}$ é a fração de hemoglobina da mistura venosa saturada com oxigênio; $\mathrm{PvO}_{2}$ é a pressão parcial de oxigênio venosa mista; 0,0031 é o coeficiente de solubilidade de Bunsen para oxigênio plasmático.

Assim como $\mathrm{CaO}_{2}, \mathrm{CvO}_{2}$ é expresso em $\mathrm{mL}$ de oxigênio por $100 \mathrm{~mL}$ ou por $\mathrm{L}$ de sangue (COLLINS et al., 2015). $\mathrm{SvO}_{2}$ apresenta valores de aproximadamente $75 \%$ em indivíduos saudáveis, o que significa uma considerável "reserva" no sistema de fornecimento de oxigênio. O nível de oxigenação do sangue venoso periférico, entretanto, pode variar dependendo do metabolismo local e do consumo de oxigênio (O' DRISCOLL et al., 2008). A PvO 2 , obtida por 
meio de catéter de termodiluição representa o oxigênio remanescente na corrente sanguínea venosa após a extração de oxigênio pelos tecidos, cujos valores, em indivíduos saudáveis, variam de 30 a $40 \mathrm{mmHg}$ (COLLINS et al., 2015).

O conteúdo capilar de oxigênio pode ser definido como (NUNN, 1987):

$$
\mathrm{Cc}^{\prime} \mathrm{O}_{2}=\mathrm{Hb}^{*} 1,34 * \mathrm{ScO}_{2}+\left(\mathrm{PAO}_{2} * 0,0031\right),
$$

No qual $H b$ é a concentração sérica de hemoglobina (g/dL); 1,34 é o volume de oxigênio carreado pela hemoglobina totalmente saturada; $\mathrm{ScO}_{2}$ é a saturação de oxigênio capilar; $\mathrm{PAO}_{2}$ é a pressão alveolar de oxigênio; 0,0031 é o coeficiente de solubilidade de Bunsen para oxigênio plasmático (COOPER et al., 2008). A saturação de oxigênio capilar é, por convenção, igual a 1,0. $\mathrm{O} \mathrm{Cc}^{\prime} \mathrm{O}_{2}$ também é expresso em $\mathrm{mL}$ de oxigênio por $100 \mathrm{~mL}$ ou por $\mathrm{L}$ de sangue (COLLINS et al., 2015).

A pressão alveolar de oxigênio $\left(\mathrm{PAO}_{2}\right)$ reflete a entrada de oxigênio para o parênquima pulmonar e sua absorção no sangue (PETERSSON; GLENNY, 2014). A equação do gás alveolar é usada para se calcular a pressão parcial de oxigênio alveolar, uma vez que não é possível coletar amostras para análises de gases diretamente dos alvéolos. A equação é útil para calcular e estimar de perto a pressão parcial de oxigênio arterial $\left(\mathrm{PaO}_{2}\right)$ dentro dos alvéolos. As variáveis da equação podem afetar a $\mathrm{PaO}_{2}$ dentro dos alvéolos em diferentes estados fisiológicos e fisiopatológicos (DIEHL; MERCAT; PESENTI, 2019). A equação do gás alveolar pode ser calculada como:

$$
\mathrm{PAO}_{2}=\left[\mathrm{FiO}_{2} *\left(\mathrm{~PB}-\mathrm{PH}_{2} \mathrm{O}\right)\right]-\left(\mathrm{PaCO}_{2} / \mathrm{R}\right),
$$

Em que $\mathrm{FiO}_{2}$ é a fração inspirada de oxigênio; $\mathrm{PB}$ é a pressão barométrica local; $\mathrm{PH}_{2} \mathrm{O}$ é a pressão de vapor de água $(47 \mathrm{mmHg}) ; \mathrm{PaCO}_{2}$ é a pressão parcial de gás carbônico arterial; $R$ é a razão de consumo de oxigênio pelo $\mathrm{CO}_{2}$ expirado, com valor estabelecido em 0,8 (NUNN, 1987).

Com a ajuda da equação do gás alveolar, a pressão parcial dentro dos alvéolos $\left(\mathrm{PAO}_{2}\right)$ pode ser calculada. O dióxido de carbono $\left(\mathrm{CO}_{2}\right)$ é uma variável muito importante na equação. A pressão de oxigênio nos alvéolos pode mudar significativamente com variações nos níveis sanguíneos e de dióxido de carbono alveolar. Se o aumento de $\mathrm{CO}_{2}$ for significativo, ele pode deslocar as moléculas de oxigênio, que causarão hipoxemia (BASHAR et al., 2018). A FiO 2 é a concentração de oxigênio na mistura de gases atmosféricos, sendo a sua concentração de $21 \%$ independentemente da altitude (PEACOCK, 1998). Muito embora esta concentração não seja equivalente ao oxigênio que participa das trocas gasosas em nível alveolar, vários fatores 
merecem consideração e são resumidos pela equação do gás alveolar, tais como PB, a pressão do vapor d'água $\mathrm{PH}_{2} \mathrm{O}$ e $\mathrm{R}$. $\mathrm{O}$ que pode ser presumido dessa relação direta é que à medida que a $\mathrm{FiO}_{2}$ aumenta, o $\mathrm{PAO}_{2}$ aumenta proporcionalmente (ALLARDET-SERVENT et al., 2009).

$\mathrm{A} \mathrm{PAO}_{2}$ é a força motriz para a difusão de oxigênio através das membranas alveolares, através das paredes dos capilares pulmonares e para o fluxo sanguíneo arteriolar e eritrócitos para transporte por todo o corpo e para os tecidos periféricos (HUANG; GOU; FU, 2019). Uma vez nos pulmões, o oxigênio se difunde através da barreira alvéolo-capilar dos alvéolos para a circulação arterial. O gradiente de difusão inicial de pressões na microcirculação surge quando a $\mathrm{PaO}_{2}$ com uma pressão mais alta é misturada com a pressão de oxigênio dentro das veias $\left(\mathrm{PvnO}_{2}\right)$ (MAYER; TRZECIAK; PURI, 2016). A taxa de difusão do oxigênio através da membrana alvéolo-capilar, além de uma eliminação mais rápida e fácil de $\mathrm{CO}_{2}$, garante que a $\mathrm{PaO}_{2}$ capilar seja quase igual à $\mathrm{PAO}_{2}$ alveolar e em condições normais (nível do mar) corresponda a 75 a $100 \mathrm{mmHg}$ (SCHEUFLER, 2004).

A pressão parcial do dióxido de carbono $\left(\mathrm{PCO}_{2}\right)$ é a medida do dióxido de carbono no sangue arterial ou venoso. Muitas vezes, serve como um marcador de ventilação alveolar adequada dentro dos pulmões (BYRNE et al., 2014). Geralmente, em condições fisiológicas normais, o valor de $\mathrm{PaCO}_{2}$ varia entre 35 a $45 \mathrm{mmHg}$, e o valor de $\mathrm{PvCO}_{2}$ apresenta valores entre 40 e $50 \mathrm{mmHg}$. Normalmente, a mensuração de $\mathrm{PCO}_{2}$ é realizada por meio de gasometria arterial; entretanto, existem outros métodos, como coleta de amostras venosa periférica, venosa central ou venosa mista (MALINOSKI et al., 2015).

A coleta de uma amostra venosa periférica por punção venosa, para teste de gasometria, não é uma alternativa confiável para substituir uma amostra arterial, pois a coleta deve evitar as alterações isquêmicas de um torniquete. Uma possibilidade de coleta de sangue venoso periférico é liberar o torniquete após a punção venosa e permitir que um minuto inteiro passe antes da coleta (CENGIZ et al., 2009). Esse processo garantirá que o $\mathrm{PCO}_{2}$ circulante seja mais preciso e também fornecerá o $\mathrm{pH}$ mais confiável. Estudos em pacientes hemodinamicamente estáveis demonstram que, em comparação, a $\mathrm{PCO}_{2}$ venosa central é aproximadamente 4 a 5 $\mathrm{mmHg}$ maior que uma amostra arterial, e a $\mathrm{PCO}_{2}$ periférica é aproximadamente 3 a $8 \mathrm{mmHg}$ maior que uma amostra arterial (WALKEY et al., 2015).

O cateter de artéria pulmonar, comumente conhecido por cateter de Swan-Ganz, permite uma mensuração precisa dos valores da mistura venosa do paciente, embora os riscos associados impossibilitem seu uso apenas para estimar a mistura venosa (SHOEMAKER; 
APPEL; D, 1988). O catéter de artéria pulmonar fornece dados hemodinâmicos que não podem ser obtidos somente por meio de avaliações clínicas (CONNORS; MCCAFFREE; GRAY, 1983), em contrapartida, há riscos inerentes à sua própria utilização (FEIN et al., 1984; HARTUNG et al., 1994), danos podem ser causados aos pacientes por má interpretação dos dados fornecidos (GNAEGI; FEIHL; PERRET, 1997; JACKA et al., 2002), e os mesmos dados podem não ser úteis no tratamento de pacientes críticos (MARIK, 2013; OOHASHI; ENDOH, 2005).

Dessa maneira, após a publicação de vários estudos na primeira década deste século que não conseguiram demonstrar um efeito benéfico desta técnica invasiva no resultado do paciente em várias situações, e também identificaram uma frequência relativamente alta de eventos adversos, algumas alternativas a seu uso começaram a ser estudadas (HARVEY et al., 2005; WALTON; HANSEN, 2018). A cateterização venosa central é mais barata e tecnicamente mais simples do que o cateterismo da artéria pulmonar, com menor potencial de complicações (LEE; BORA, 2020). Embora o sangue venoso misto seja o ideal para cálculo do shunt pulmonar, a necessidade de usar um catéter de termodiluição para acessar o sangue da artéria pulmonar é muito mais laboriosa se comparada ao uso do cateter venoso central, situado na veia cava superior, com acesso ao átrio direito (IODICE et al., 2014; WARNER et al., 2009).

Apesar de existirem poucos relatos da relação entre as duas técnicas na medicina humana, em condições fisiológicas, as amostras de sangue venosas centrais predizem as venosas mistas razoavelmente bem, geralmente $2-5 \%$ menos, em grande parte devido aos efeitos de alta captação cerebral de oxigênio e alta de fluxo sanguíneo renal (HARTOG; BLOOS, 2014). Assim, em humanos, o sangue venoso central pode, de fato, atuar como substituto do sangue venoso misto em pacientes hígidos (DUECK et al., 2005; REINHART, 2004). Tomando como exemplo, a saturação venosa central $\left(\mathrm{ScvO}_{2}\right)$, um limite mínimo de $70 \%$ foi sugerido com base em trabalhos experimentais e na experiência da medicina humana, uma vez que sua mensuração é muito utilizada como índice de oxigenação tecidual (BEEST et al., 2011). Usando esse ponto de corte em um estudo, 11 de um total de 30 cães gravemente doentes $\left(\mathrm{ScvO}_{2}<70 \%\right)$ foram suspeitos de ter choque oculto, apesar da normalização da frequência cardíaca, pressão arterial, atividade cerebral e parâmetros de perfusão, sugerindo doença grave persistente, apesar dos achados clínicos sugerirem o contrário (YOUNG et al., 2014).

Futuros estudos veterinários de oxigenação venosa devem incluir em avaliações de monitoramento contínuo, a sensibilidade e especificidade da $\mathrm{ScvO}_{2}$ para identificar problemas clinicamente importantes e suas adequações (WALTON; HANSEN, 2018). Assim sendo, o 
sangue venoso central está altamente correlacionado e pode substituir o sangue venoso misto na análise da função pulmonar e oxigenação tecidual (GEHRCKE; REGALIN; PADILHA, 2017). Ainda, com a possibilidade de se utilizar sangue venoso central, o shunt pulmonar pode ser calculado sem que haja prejuízos à saúde dos pacientes (ARAOS et al., 2012). 


\section{REFERÊNCIAS}

AKMAL, A. H.; HASAN, M.; MARIAM, A. The incidence of complications of central venous catheters at an intensive care unit. Annals of Thoracic Medicine, v. 2, n. 2, p. 61-63, 2007.

ALLARDET-SERVENT, J. et al. FiO2 and acute respiratory distress syndrome definition during lung protective ventilation. Critical Care Medicine, v. 37, n. 1, p. 202-207, 2009. ARAOS, J. D. et al. Use of the oxygen content-based index, Fshunt, as an indicator of pulmonary venous admixture at various inspired oxygen fractions in anesthetized sheep.

American Journal Of Veterinary Research, v. 73, n. 12, p. 2013-2020, 2012.

ARAÚJO, M. A. et al. Cardiopulmonary effects of reverse Trendelenburg position at $5^{\circ}$ and $10^{\circ}$ in sevoflurane-anesthetized steers. Veterinary Anaesthesia and Analgesia, v. 44, n. 4, p. 854-864, 2017.

BARBONI, B. et al. Synthetic Bone Substitute Engineered with Amniotic Epithelial Cells Enhances Bone Regeneration after Maxillary Sinus Augmentation. PLoS ONE, v. 8, n. 5, 2013.

BARRY, J. S.; ANTHONY, R. V. The pregnant sheep as a model for human pregnancy. Theriogenology, v. 69, n. 1, p. 55-67, 2008.

BASHAR, F. R. et al. Comparison of non-invasive to invasive oxygenation ratios for diagnosing acute respiratory distress syndrome following coronary artery bypass graft surgery : a prospective derivation-validation cohort study. v. 8, p. 1-8, 2018.

BEEST, P. VAN et al. Clinical review : use of venous oxygen saturations as a goal - a yet unfi nished puzzle. p. 1-9, 2011.

BIZOUARN, P.; BLANLOEILAND, Y.; PINAUD, M. Comparison between oxygen consumption calculated by Fick' s principle using a continuous thermodilution technique and measured by indirect calorimetry. British Journal of Anaesthesia, v. 75, p. 719-723, 1995.

BLAZE, C. A.; LEBLANC, P. H.; ROBINSON, N. E. Effect of withholding feed on ventilation and the incidence of regurgitation during halothane anesthesia of adult cattle. American Journal Of Veterinary Research, v. 49, p. 2126-2129, 1988.

BRAZZI, L. et al. General Design and Results of the Feasibility Phase of a Multicenter, Randomized Trial of Three Different Hemodynamic Monitoring Approaches and Two of Techniques in the Treatment Critically Ill Patients. Controlled Clinical Trials, v. 16, p. 74 87, 1995.

BRIGANTI, A. et al. Accuracy of different oxygenation indices in estimating intrapulmonary shunting at increasing infusion rates of dobutamine in horses under general anaesthesia.

Veterinary Journal, v. 204, n. 3, p. 351-356, 2015.

BRISMAR, B. et al. Pulmonary densities during anesthesia with muscular relaxation - a proposal of atelectasis. Anesthesiology, v. 62, n. 4, p. 422-428, 1985.

BYRNE, A. N. L. et al. Peripheral venous and arterial blood gas analysis in adults : are they comparable? A systematic review and meta-analysis. v. 2013, n. November 2013, 2014. CALASANS-MAIA, M. D. et al. The rabbit as an animal model for experimental surgery. Acta Cirurgica Brasileira, v. 24, n. 4, p. 325-328, 2009.

CARBONE, L. Pain in laboratory animals: The ethical and regulatory imperatives. PLoS ONE, v. 6, n. 9, 2011.

CARROLL, G. L.; HARTSFIELD, S. M. General anesthetic techniques in ruminants. The Veterinary clinics of North America. Food animal practice, v. 12, n. 3, p. 627-661, 1996. CENGIZ, M. et al. Influence of tourniquet application on venous blood sampling for serum chemistry, hematological parameters, leukocyte activation and erythrocyte mechanical properties. v. 47, n. 6, p. 769-776, 2009.

CLAES, L. E. et al. Effects of mechanical factors on the fracture healing process. Clinical Orthopaedics and Related Research, n. 355 SUPPL., 1998. 
CLARKE, K. W. Desflurane and sevoflurane: New volatile anesthetic agents. Veterinary

Clinics of North America - Small Animal Practice, v. 29, n. 3, p. 793-810, 1999.

COLLINS, J.-A. et al. Relating oxygen partial pressure, saturation and content : the haemoglobin - oxygen dissociation curve. Breathe, v. 11, n. 3, p. 194-201, 2015.

CONNORS, A. F.; MCCAFFREE, D. R.; GRAY, B. A. Evaluation of right-heart catheterization in the critically ill patient without acute myocardial infarction. The New England Journal of Medicine, v. 308, n. 5, p. 263-267, 1983.

COOPER, C. B. et al. Venous Admixture in COPD : Pathophysiology and. n. December, p. 376-381, 2008.

CORONA, T. M.; AUMANN, M. Ventilator waveform interpretation in mechanically ventilated small animals. Journal of Veterinary Emergency and Critical Care, v. 21, n. 5, p. 496-514, 2011.

DIEHL, J. L.; MERCAT, A.; PESENTI, A. Understanding hypoxemia on - back to the alveolar gas equation. Intensive Care Medicine, v. 45, n. 2, p. 255-256, 2019.

DRISCOLL, B. R. O. et al. BTS guideline for emergency oxygen use in adult patients. v. 63, n. fig 2, 2008.

DUECK, M. H. et al. Trends but Not Individual Values of Central Venous Oxygen Saturation Agree with Mixed Venous Oxygen Saturation during Varying Hemodynamic Conditions. n. 2, p. 249-257, 2005.

DUECK, R.; RATHBUN, M.; GREENBURG, G. Lung volume and VA/Q distribution response to intravenous versus inhalalation anesthesia in sheep. Anesthesiology, v. 61, p. 5565, 1984.

DUGGAN, M.; KAVANAGH, B. P. Pulmonary Atelectasis: A Pathogenic Perioperative Entity. Anesthesiology, v. 102, n. 4, p. 838-854, 2005.

EGERMANN, M.; GOLDHAHN, J.; SCHNEIDER, E. Animal models for fracture treatment in osteoporosis. Osteoporosis International, v. 16, n. SUPPL. 2, p. 129-138, 2005.

ELLIOTT, R. H.; STRUNIN, L. Hepatotoxicity of volatile anaesthetics. British Journal of Anaesthesia, v. 70, n. 3, p. 339-348, 1993.

EWING, K. K. Anesthesia techniques in sheep and goats. The Veterinary clinics of North America. Food animal practice, v. 6, n. 3, p. 759-778, 1990.

FAGUNDES, D. J.; TAHA, M. O. Modelo animal de doença: critérios de escolha e espécies de animais de uso corrente. Acta Cirurgica Brasileira, v. 19, n. 1, p. 59-65, 2004.

FEIN, A. M. et al. Is pulmonary artery catheterization necessary for the diagnosis of pulmonary edema? American Review of Respiratory Diseases, v. 129, p. 1006-1009, 1984. FLANAGAN, T. C. et al. In Vivo Remodeling and Structural Characterization of FibrinBased Tissue-Engineered Heart Valves in the Adult Sheep Model. Tissue Engineering Part A, v. 15, n. 10, p. 2965-2976, 2009.

FRISKEN, K. W. et al. A study of titanium release into body organs following the insertion of single threaded screw implants into the mandibles of sheep. Australian Dental Journal, v. 47, n. 3, p. 214-217, 2002.

FUJIMOTO, J. I.; LENEHAN, T. M. The Influence of Body Position on the Blood Gas and Acid-base Status of Halothane-anesithetized Sheep. Veterinary Surgery, v. 14, n. 2, p. 169$172,1985$.

FUJINO, Y. et al. Repetitive high-pressure recruitment maneuvers required to maximally recruit lung in a sheep model of acute respiratory distress syndrome. Critical Care Medicine, v. 29, n. 8, p. 1579-1586, 2001.

GALATOS, A. D. Anesthesia and Analgesia in Sheep and Goats. Veterinary Clinics of North America - Food Animal Practice, v. 27, n. 1, p. 47-59, 2011.

GARAY, S.; KAMELAR, D. Pathophysiology of trauma-associated respiratory failure. In: HOOD, R. M.; BOYD, A. D.; CULLIFORD, A. T. (Eds.). . Thoracic Trauma. Philadelphia: 
Saunders, 1989. p. 328-332.

GEHRCKE, M. I.; REGALIN, D.; PADILHA, V. S. Arterial, Mixed Venous or Central Venous Hemogasometry and End Tidal CO 2 in Dogs under Different Hemodynamic States. 2017.

GENÇCELEP, M.; ATASOY, N.; TAS, A. The effects of inhalation anaesthetics ( halothane and isoflurane ) on certain clinical and haematological parameters of sheep. v. 53, p. 157-160, 2004.

GILBERT, R.; KEIGHLEY, J. The arterial-alveolar oxygen tension ratio. An index of gas exchange applicable to varying inspired oxygen concentrations. Am Rev Respir Dis, v. 18, p. 1043-1048, 1974.

GNAEGI, A.; FEIHL, F.; PERRET, C. Intensive care physicians' insufficient knowledge of right-heart catheterization at the bedside: Time to act? Critical Care Medicine, v. 25, n. 2, p. 213-220, 1997.

GRAY, P. R.; MCDONELL, W. N. Anaesthesia in goats and sheep. Part II. General anesthesia. Compendium of Continuing Education for the Practicing Veterinarian Supplement, v. 8, p. 127-135, 1986.

HALL, L. W.; CLARKE, K. W.; TRIM, C. M. Veterinary Anaesthesia. 10th. ed. London: W. B. Saunders, 2001.

HARTOG, C.; BLOOS, F. Best Practice \& Research Clinical Anaesthesiology Venous oxygen saturation. Best Practice \& Research Clinical Anaesthesiology, v. 28, n. 4, p. 419428, 2014.

HARTUNG, E. J. et al. Severe Air Embolism Caused by a Pulmonary Artery Introducer Sheath. Anesthesiology, v. 80, p. 1402-1403, 1994.

HARVEY, S. et al. Assessment of the clinical effectiveness of pulmonary artery catheters in management of patients in intensive care ( PAC-Man ): a randomised controlled trial. v. 366, 2005.

HICKEY, R. F. et al. Effects of halotane anesthesia on functional residual capacity and alveolar-arterial oxygen tension difference. Anesthesiology, v. 38, n. 1, p. 20-24, 1973. HIKASA, Y. et al. Comparisons of sevoflurane, isoflurane, and halothane anesthesia in spontaneously breathing cats. Veterinary Surgery, v. 25, n. 3, p. 234-243, 1996.

HILLMAN, N. H. et al. Brief, large tidal volume ventilation initiates lung injury and a systemic response in fetal sheep. American Journal of Respiratory and Critical Care Medicine, v. 176, n. 6, p. 575-581, 2007.

HOPSTER, K.; KASTNER, S. B. R.; ROHN, K. Intermittent positive pressure ventilation with constant positive end-expiratory pressure and alveolar recruitment manoeuvre during inhalation anaesthesia in horses undergoing surgery for colic, and its influence on the early recovery period. Vet Anaesth Analg, v. 38, p. 169-177, 2011.

HUANG, F.; GOU, Z.; FU, Y. Preliminary evaluation of a predictive controller for a rotary blood pump based on pulmonary oxygen gas exchange. v. 233, n. 2, p. 267-278, 2019.

IODICE, F. G. et al. Fiberoptic monitoring of central venous oxygen saturation ( PediaSat ) in small children undergoing cardiac surgery: Continuous is not continuous Fiberoptic monitoring of central venous oxygen saturation ( PediaSat ) in small children undergoing cardiac surgery : continuous is not continuous [ v2; ref status : indexed, http://f1000r.es/394 ]. n. December, 2014.

IVANY, J. M.; MUIR, W. W. Farm animal anesthesia. In: FUBINI, S. L.; DUCHARME, N. G. (Eds.). . Farm animal surgery. 2nd. ed. Saint Louis: W. B. Saunders, 2004. p. 97-112. JACKA, M. J. et al. Pulmonary artery occlusion pressure estimation: How confident are anesthesiologists? Critical Care Medicine, v. 30, n. 6, p. 1197-1203, 2002.

KLEIN, L.; FISHER, N. Cardiopulmonary effects of restraint in dorsal recumbency on awake cattle. American Journal Of Veterinary Research, v. 49, p. 1605-1608, 1988. 
LE, M. H. T. et al. Adjunctive buccal and palatal corticotomy for adult maxillary expansion in an animal model. Korean Journal of Orthodontics, v. 48, n. 2, p. 98-106, 2018.

LEE, C. P.; BORA, V. Anesthesia Monitoring Of Mixed Venous Saturation. p. 1-8, 2020. LERNER, Z. F. et al. Modulating tibiofemoral contact force in the sheep hind limb via treadmill walking: Predictions from an opensim musculoskeletal model. Journal of Orthopaedic Research, v. 33, n. 8, p. 1128-1133, 2015.

LORING, S. H.; BUTLER, J. P. Gas exchange in body cavities. In: FARHI, L. E.; TENNEY, S. M. (Eds.). . Handbook of Physiology. Bethesda: American Physiological Society, 1987. p. 283-295.

LOVERING, A. T. et al. transpulmonary shunting into the general circulation : An update. v. 41, n. 0 2, p. 1-20, 2015.

LUMB, A. B. Nunn's Applied Respiratory Physiology. 5th. ed. Oxford: ButterworthHeinemann, 2000.

MALINOSKI, D. J. et al. Correlation of Central Venous and Arterial Blood Gas

Measurements in Mechanically Ventilated Trauma Patients. v. 140, 2015.

MARIK, P. E. Obituary: Pulmonary artery catheter 1970 to 2013. Annals of Intensive Care, v. 3, n. 1, p. 1-6, 2013.

MARTINI, L. et al. Sheep Model in Orthopedic Research: A Literature Review. Comparitive Medicine, v. 51, n. 4, p. 292-299, 2001.

MAYER, K.; TRZECIAK, S.; PURI, N. K. Assessment of the adequacy of oxygen delivery. p. 437-443, 2016.

MCMILLEN, C. The sheep - an ideal model for biomedical research? Anzccart News, v. 14, n. 2, p. 1-4, 2001.

MEEUSEN, E. N. et al. Sheep as a model species for the study and treatment of human asthma and other respiratory diseases. Drug Discovery Today: Disease Models, v. 6, n. 4, p. 101-106, 2009.

MEYER, H. et al. Cardiopulmonary effects of dorsal recumbency and high-volume caudal epidural anaesthesia with lidocaine or xylazine in calves. Veterinary Journal, v. 186, n. 3, p. 316-322, 2010.

MOHAMADNIA, A. R.; HUGHES, G.; CLARKE, K. W. Maintenance of anaesthesia in sheep with isoflurane, desflurane or sevoflurane. Veterinary Record, v. 163, n. 7, p. 210 215, 2008.

MURAKAMI, K. et al. A novel animal model of sepsis after acute lung injury in sheep.

Critical Care Medicine, v. 30, n. 9, p. 2083-2090, 2002.

MUTOH, T. et al. Cardiopulmonary effects of sevoflurane, compared with halothane, enflurane, and isoflurane, in dogs. American Journal Of Veterinary Research, v. 58, n. 8, p. 885-890, 1997.

NUNN, J. F.; HILL, D. W. Respiratory dead space and arterial to end-tidal CO2 tension difference in anesthetized man. Journal of Applied Physiology, v. 15, n. 3, p. 383-389, 1960.

OLIVEN, A.; ABINADER, E.; BURSZTEIN, S. Influence of varying inspired oxygen tensions on the pulmonary venous admixture (shunt) of mechanically ventilated patients.

Critical Care Medicine, v. 8, p. 99-101, 1980.

OOHASHI, S.; ENDOH, H. Does central venous pressure or pulmonary capillary wedge pressure reflect the status of circulating blood volume in patients after extended transthoracic esophagectomy? Journal of Anesthesia, v. 19, n. 1, p. 21-25, 2005.

PEACOCK, A. J. Oxygen at high altitude. p. 1063-1066, [s.d.].

PEARCE, A. I. et al. Animal models for implant biomaterial research in bone: A review.

European Cells and Materials, v. 13, n. 0, p. 1-10, 2007.

PEARSE, R. M.; RHODES, A. Mixed and Central Venous Oxygen Saturation. 1970. 
PERILLI, V. et al. Comparison of positive end-expiratory pressure with reverse Trendelenburg position in morbidly obese patients undergoing bariatric surgery: Effects on hemodynamics and pulmonary gas exchange. Obesity Surgery, v. 13, n. 4, p. 605-609, 2003. PETERSSON, J.; GLENNY, R. W. Gas exchange and ventilation-perfusion relationships in the lung. European Respiratory Journal, v. 44, n. 4, p. 1023-1041, 2014.

PORCELLI, R. J. Pulmonary hemodynamics. In: Treatise on Pulmonary Toxicology. Vol. 1: Comparative Biology of the Normal Lung. [s.l: s.n.]. p. 241-270.

REES, S. E. et al. Assessing pulmonary congestion in left sided heart failure using pulmonary gas exchange parameters. IEEE Engineering in Medicine and Biology Society, p. 435-438, 2003.

REHDER, K. et al. Effects of general anesthesia, muscle paralysis, and mechanical ventilation on pulmonary nitrogen clearance. Anesthesiology, v. 35, n. 6, p. 591-601, 1971.

REHDER, K. et al. Ventilation-perfusion relationship in young healthy awake and anesthetized-paralyzed man. Journal of applied physiology: respiratory, environmental and exercise physiology, v. 47, n. 4, p. 745-753, 1979.

REINHART, K. Continuous central venous and pulmonary artery oxygen saturation monitoring in the critically ill. p. 1572-1578, 2004.

ROBINSON, N. E. The respiratory system. In: MUIR, W. W.; HUBBELL, J. A. E. (Eds.). . Equine Anesthesia: Monitoring and Emergency Therapy. 2nd. ed. St Louis, MO: Saunders Elsevier, 2009. p. 11-36.

ROSA, L. D. A. MODALIDADES VENTILATÓRIAS EM OVINOS Dissertação apresentada ao programa de pós- graduação em Ciência Animal da Universidade do Estado de Santa Catarina - UDESC , como requisito para a obtenção do título de Mestre em Ciência Animal . Orientador : Prof . Dr . Nilson Oleskovicz LAGES - SC. 2017.

ROTHEN, H. U. et al. Atelectasis and pulmonary shunting during induction of general anaesthesia - Can they be avoided? Acta Anaesthesiologica Scandinavica, v. 40, n. 5, p. 524-529, 1996.

SARTORETTO, S. C. et al. Sheep As an Experimental Model for Biomaterial Implant Evaluation. Acta Ortopédica Brasileira, v. 24, n. 5, p. 262-266, 2016.

SCHEUFLER, K. Tissue oxygenation and capacity to deliver O 2 do the two go together? $\mathrm{v}$. 31, p. 45-54, 2004.

SHOEMAKER, V. C.; APPEL, P. L.; D, T. L. A. I. Prospective Trial of Supranormal Values of Survivors as Therapeutic Goals in High- Risk Surgical Patients *. 1988.

SIDDIQI, A. et al. One-piece zirconia ceramic versus titanium implants in the jaw and femur of a sheep model: A pilot study. BioMed Research International, v. 2016, 2016.

SINGH, I.; PINSKY, M. R. Heart-Lung Interactions. First Edit ed. [s.l.] Elsevier Inc., 2008. STAUB, N. C. Alveolar-arterial oxygen tension gradient due to diffusion. Journal of applied physiology (Bethesda, Md. : 1985), v. 18, p. 673-680, 1963.

STEFFEY, E. P. Some characteristics of ruminants and swine that complicate management of general anesthesia. The Veterinary clinics of North America. Food animal practice, v. 2, n. 3, p. 507-516, 1986.

STEFFEY, E. P.; MAMA, K. R.; BROSNAN, R. J. Inhalation anesthetics. In: GRIMM, K. A. et al. (Eds.). . Veterinary Anesthesia and Analgesia: The Fifth Edition of Lumb and Jones. 5th. ed. [s.1.] Wiley Blackwell, 2015. p. 297-323.

TATARA, A. M. et al. Reconstruction of large mandibular defects using autologous tissues generated from in vivo bioreactors. Acta Biomaterialia, v. 45, p. 72-84, 2016.

TRANQUILLI, W. J. Techniques of inhalation anesthesia in ruminants and swine.

Veterinary Clinics of North America. Food Animal Practice, v. 2, n. 3, p. 593-619, 1986. TRIM, C. M. Sedation and general anesthesia in ruminants. Calif Vet, v. 35, p. 29-36, 1981. TURNER, A. S. Experiences with sheep as an animal model for shoulder surgery: Strengths 
and shortcomings. Journal of Shoulder and Elbow Surgery, v. 16, n. 5 SUPPL., p. 158$163,2007$.

VALVERDE, A.; DOHERTY, T. J. Anesthesia and analgesia in ruminants. In: FISH, R. et al. (Eds.). . Anesthesia and analgesia in laboratory animals. 2nd. ed. London: Academic Press, 2008. p. 385-411.

WAGNER, A. E.; MUIR, W. W.; GROSPITCH, B. J. Cardiopulmonary effects of position in conscious cattle. American Journal Of Veterinary Research, v. 51, p. 7-10, 1990.

WAGNER, P. D. et al. Continuous distributions of ventilation perfusion ratios in normal subjects breathing air and 100\% O2. Journal of Clinical Investigation, v. 54, n. 1, p. 54-68, 1974.

WALKEY, A. J. et al. The Accuracy of the Central Venous Blood Gas for Acid - Base Monitoring. v. 25, n. 2, p. 104-110, 2015.

WALLEY, K. R. Use of Central Venous Oxygen Saturation to Guide Therapy. Concise Clinical Review, v. 184, p. 514-520, 2011.

WALSH, W. R. et al. Effects of Low-Intensity Pulsed Ultrasound on Tendon-Bone Healing in an Intra-articular Sheep Knee Model. Arthroscopy - Journal of Arthroscopic and Related Surgery, v. 23, n. 2, p. 197-204, 2007.

WALTON, R. A. L.; HANSEN, B. D. Venous oxygen saturation in critical illness. v. 28, n. 5, p. 387-397, 2018.

WARNER, D. S. et al. VI REVIEW ARTICLES Role of Central and Mixed Venous Oxygen Saturation Measurement in Perioperative Care. n. 3, p. 649-656, 2009.

WATANABE, M.; FONSECA, C. D.; VATTIMO, M. F. F. Instrumental and ethical aspects of experimental research with animal models. Revista da Escola de Enfermagem da

Universidade de São Paulo, v. 48, n. 1, p. 177-183, 2014.

WEISKOPF, R. B.; SEVERINGHAUS, J. W. Diffusing capacity of the lung for CO in man during acute acclimation to 14,246 ft. Journal of Applied Physiology, v. 32, n. 3, p. 285289, 1972.

WEST, J. B. Ventilation-perfusion relationships. Am Rev Respir Dis, v. 116, p. 919-943, 1977.

WHITE, K.; TAYLOR, P. Anaesthesia in sheep. In Practice, v. 22, n. 3, p. 126-128,131$133,135,2000$.

YOUNG, B. C. et al. Decreased central venous oxygen saturation despite normalization of heart rate and blood pressure post shock resuscitation in sick dogs. v. 24, n. 2, p. 154-161, 2014. 


\title{
5 CAPÍTULO 2 - INFLUENCE OF RECUMBENCY ON THE PULMONARY SHUNT IN SEVOFLURANE-ANAESTHETIZED SHEEP
}

Enviado para publicação em 29/03/2021 para a revista "Ciência Rural”, sob o protocolo CR2021-0251.

\begin{abstract}
This study assessed the ventilatory changes and pulmonary shunt in sevoflurane-anaesthetised sheep underwent different recumbencies. Seven female sheep were premedicated with 0.1 mg. $\mathrm{kg}^{-1}$ butorfanol and subsequently administered a combination of $3 \mathrm{mg} . \mathrm{kg}^{-1}$ ketamine and 0.5 mg.kg-1 midazolam. Animals were intubated and maintained on sevoflurane anaesthesia with pressure-controlled ventilation ( $12 \mathrm{~cm} \mathrm{H}_{2} \mathrm{O}$ peak inspiratory pressure) and $f$ of $10 \mathrm{mpm}$. During the anaesthetic procedure, animals underwent three different recumbencies: dorsal, left lateral, or right lateral positions. Treatments lasted $120 \mathrm{~min}$ with a 48 -h washout period in between the treatments. Arterial and central venous blood samples were withdrawn immediately after anaesthetic induction ( 0 minute), and at 60 and 120 minutes from induction. Obtained values were used to calculate pulmonary shunt fraction (V/Q), $\mathrm{CaO}_{2}, \mathrm{CcvO}_{2}$, and $\mathrm{CcO}_{2}$ accordingly. Results showed that V/Q greatly decreased from 0 to $120 \mathrm{~min}$ in all the groups (dorsal: 69.3\% to $27.3 \%$; left lateral: $59.1 \%$ to $25.0 \%$; right lateral: $67.2 \%$ to $32.4 \%$ ). $\mathrm{CaO}_{2}, \mathrm{CcvO}_{2}$ and $\mathrm{CcO}_{2}$ improved over time points, with no difference among treatments. $\mathrm{PaO}_{2}$ and $\mathrm{PAO}_{2}$ showed higher values for 60 and 120 min compared to the 0 min value in all groups, with no differences among treatments as well. $\mathrm{PaCO}_{2}(50.5 \pm 12.0 \mathrm{mmHg}$ and $50.6 \pm 7.7 \mathrm{mmHg})$ and $\mathrm{ETCO}_{2}$ $(42.5 \pm 10.4 \mathrm{mmHg}$ and $41.6 \pm 6.8 \mathrm{mmHg}$ ) were higher in the right and left lateral groups, respectively, than those in the dorsal group at $120 \mathrm{~min}$. Pressure-controlled ventilation improved gas exchanges in sheep, thereby reducing pulmonary shunt. In conclusion, recumbency did not interfere with pulmonary shunt.
\end{abstract}

Key words: sevoflurane, sheep, pulmonary shunt, mechanical ventilation. 


\section{RESUMO}

O presente estudo avaliou o impacto de diferentes decúbitos em ovelhas anestesiadas com sevoflurano, priorizando alterações ventilatórias e de shunt pulmonar. Sete ovelhas foram prémedicadas com 0.1 mg.kg-1 de butorfanol e induzidas à anestesia com 3 mg.kg-1 de cetamina e 0.5 mg.kg-1 de midazolam. Os animais foram intubados e mantidos em anestesia por sevofluorano, em ventilação mecânica controlada por pressão, com pico inspiratório em $12 \mathrm{~cm}$ $\mathrm{H}_{2} \mathrm{O}$ e $f$ de $10 \mathrm{mpm}$, sendo mantidos por 120 minutos. Durante esse período os animais foram submetidos a três tratamentos: decúbitos dorsal, lateral esquerdo ou lateral direito, com intervalo de 48 horas entre eles. Foram coletadas amostras de sangue arterial e venoso para análises de gases sanguíneos e eletrólitos, imediatamente após indução anestésica (minuto 0), decorridos 60 minutos da indução e após 120 minutos da indução. Os valores obtidos foram utilizados para calcular a fração de shunt pulmonar (V/Q), $\mathrm{CaO}_{2}, \mathrm{CcvO}_{2} \mathrm{e} \mathrm{CcO}_{2}$. Os resultados mostraram que V/Q diminuiu expressivamente de 0 a 120 minutos em todos os grupos (dorsal: $69,3 \%$ para $27,3 \%$; lateral esquerdo: $59,1 \%$ para $25,0 \%$; lateral direito: $67,2 \%$ para $32,4 \%$ ). Os índices de $\mathrm{CaO}_{2}, \mathrm{CcvO}_{2}$ e $\mathrm{CcO}_{2}$ melhoraram ao longo do tempo, sem diferença entre tratamentos. $\mathrm{PaO}_{2}$ e $\mathrm{PAO}_{2}$ apresentaram valores maiores, em todos os grupos, nos minutos 60 e 120 em comparação ao momento 0 , não havendo diferenças entre tratamentos. $\mathrm{PaCO}_{2}$ $(50.5 \pm 12.0 \mathrm{mmHg}$ e $50.6 \pm 7.7 \mathrm{mmHg})$ e $\mathrm{ETCO}_{2}(42.5 \pm 10.4 \mathrm{mmHg}$ e $41.6 \pm 6.8 \mathrm{mmHg})$ apresentaram maiores valores nos grupos laterais direito e esquerdo, respectivamente, em comparação ao grupo dorsal ao final do procedimento. Conclui-se que o decúbito não interferiu na formação de shunt pulmonar.

Palavras-chave: sevoflurano, ovinos, shunt pulmonar, ventilação mecânica. 


\subsection{INTRODUCTION}

Sheep has been one of the most used experimental models in biomedical research, since it seems to mimic human clinical conditions. Some characteristics make this species scientifically acceptable, such as easy handling, bone composition, and remodelling like in humans (EGERMANN et al., 2005). It is also suitable for studying the main physiological systems such as cardiovascular, endocrine, respiratory, renal, and reproductive systems (MCMILLEN, 2001).

In large surgeries, inhalation anaesthesia is recommended; however, some issues in sheep inhalation anaesthesia must be considered. The rumen occupies about three quarters of the abdominal cavity in sheep and its proximity to diaphragm can interfere with ventilation during the procedure (EWING, 1990) resulting in hypoxaemia and hypercapnia when they are positioned in lateral or dorsal recumbency (BLAZE et al., 1988). Furthermore, these animals produce a large amount of saliva to balance ruminal $\mathrm{pH}$, and inadequate fasting can lead to regurgitation, obstruction, aspiration, asphyxia and pneumonia in the postoperative period (TRANQUILLI, 1986).

Ventilation impairment during general anaesthesia can lead to pulmonary ventilationperfusion mismatch (PETERSSON \& GLENNY, 2014). Impaired gas exchanges during inhalation anaesthesia have been related to inspired gases ventilation mismatch, reduced lung volume, airway obstruction, and pulmonary hypoxia (GALATOS, 2011). Pulmonary shunt is defined as the pathological condition in which the pulmonary alveoli are normally perfused, however, ventilation fails to supply the perfused region (LOVERING et al., 2015).

Reduced lung volume is the main cause of shunt in sheep undergoing inhalation anaesthesia (DUECK; RATHBUN; GREENBURG, 1984), and it can be worse, depending on the recumbency (GALATOS, 2011). The diaphragmatic compression that occurs due to rumen distension, by reduced organ activity or atony, substantially reduces lung compliance, especially when animals are placed in lateral or dorsal recumbencies (MEYER et al., 2010). Right lateral recumbency is associated with potentiation of diaphragmatic compression's deleterious effects on pulmonary ventilation, once $\mathrm{PaO}_{2}$ values decreased from $333 \mathrm{mmHg}$ to $299 \mathrm{mmHg}$ at 90 minutes of anaesthesia (FUJIMOTO \& LENEHAN, 1985). In dorsal recumbency with reverse Trendelenburg position, there is no improvement in respiratory function or worsening in steers cardiopulmonary function, as shunt fractions were significantly increased at $10^{\circ}$ reverse Trendelenburg position (39.4 $\pm 4.5 \%$ ) (ARAÚJO et al., 2017). Conversely, better ventilatory stability and tissue oxygenation in mechanically-ventilated sheep has been proposed (FUJINO et al., 2001). 
The importance of studying different recumbencies lies in different possible impacts on sheep anaesthesia, potentiating ventilation-perfusion mismatches. Effects of dorsal, right and left lateral recumbencies on blood gas analysis and acid-base status have been assessed in sheep, however, pulmonary shunt was not addressed so far (FUJIMOTO; LENEHAN, 1985). Effects of recumbency and lung recruitment maneuvers on haemodynamic and blood gas analysis in sheep, under pressure-controlled ventilation, have also been recorded. However, data were not correlated to recumbency nor to pulmonary shunt (FUJINO et al., 2001). Thus, this study aimed to verify if different recumbencies impact sheep inhalation anaesthesia. We hypothesized that different recumbencies determine the pulmonary shunt magnitude.

\subsection{MATERIALS AND METHODS}

Seven female adult half-blooded Dorper sheep, body weight range 40 to $60 \mathrm{~kg}$, aging over 5 years old, were used in this study. Health status was checked by clinical assessment and laboratory tests (complete blood cell count, gama-glutamyltransferase, aspartateaminotransferase, urea, and creatinine). The animals were subjected to three different treatments, namely: dorsal, right lateral, and left lateral recumbency, each one corresponding to an experimental group. All sheep were submitted to all treatments, which lasted $120 \mathrm{~min}$, and recumbency selection order was defined by balanced randomisation, to avoid treatment sequence repetition. Washout period was at least $48 \mathrm{~h}$.

Food was withheld $12 \mathrm{~h}$ preceding the experimental days, without water restriction. A 7-F $20 \mathrm{~cm}$ longer central venous catheter was introduced in the jugular vein and fixed into the animals $24 \mathrm{~h}$ before the first assessment day, following the manufacturer's instructions. For the procedure, animals were given a premedication with $0.1 \mathrm{mg} \cdot \mathrm{kg}^{-1}$ butorfanol IM. After a 10minute period, sheep were administered a combination of $3 \mathrm{mg} \cdot \mathrm{kg}^{-1}$ ketamine and $0.5 \mathrm{mg} \cdot \mathrm{kg}^{-1}$ midazolam IV.

On experimental days, sheep were given the same anaesthetic protocol as mentioned before. Right away after induction, arterial and central venous blood samples were collected (1 $\mathrm{mL}$ each, heparinized syringes) from the auricular artery and jugular vein puncture, for blood gas analysis. Thereon, animals were orally intubated with an appropriate cuffed tracheal tube, placed on the drawn recumbency (dorsal, right lateral or left lateral), and connected to a circle circuit system. Anaesthesia maintenance was performed with a calibrated sevoflurane vaporizer, in $95 \pm 2 \% \mathrm{O}_{2}$, at a flow rate of $1.5 \mathrm{~L} \cdot \mathrm{min}^{-1}$.

Muscle blockade was done by $0.12 \mathrm{mg} \cdot \mathrm{kg}^{-1}$ rocuronium, IV, and all sheep were mechanically ventilated in pressure-controlled ventilation (PCV) mode with a $12 \mathrm{~cm} \mathrm{H}_{2} \mathrm{O}$ peak 
inspiratory pressure in a zero end-expiratory pressure, $f$ adjusted to $10 \mathrm{mpm}$ and inspiratoryto-expiratory ratio 1:2 (WATO EX-20, Shenzhen Mindray Bio-Medical Electronics Co., Ltd.). When necessary, animals were administered a quarter of the rocuronium loading dose. Endtidal sevoflurane concentration (FE'Sevo) and end-tidal carbon dioxide concentration (FE'CO2) were determined using sidestream capnography, obtained by anaesthetic gas analyzer. (FE'Sevo) was maintained at a level of $2.03 \pm 0.11 \%$ during the entire procedure. A multiparameter monitor (Digicare ${ }^{\circledR}$ LifeWindow ${ }^{\mathrm{TM}}$ Lite, Digicare Biomedical Technology, Boynton Beach, Florida, USA) was used to assess: Heart rate (HR) obtained by DII derivation electrocardiogram; hemoglobin oxygen saturation $\left(\mathrm{SpO}_{2}\right)$ obtained by pulse oximetry; arterial blood pressures were measured invasively, by puncturing the auricular artery with a $22 \mathrm{G}$ catheter; and body temperature was measured by an oesophageal thermometer and obtained in Celsius. Ringer's lactate solution was infused intravenously to all sheep at a rate of $3 \mathrm{~mL} \cdot \mathrm{kg}^{-}$ ${ }^{1} \cdot \mathrm{h}^{-1}$.

Arterial and venous blood gas analysis were performed immediately after induction (0 minute), $60 \mathrm{~min}$ and $120 \mathrm{~min}$ after induction, from the auricular artery and central venous catheter for immediate analysis (CG8+, Abbott®, São Paulo, Brazil). Potential of hydrogen $(\mathrm{pH})$, carbon dioxide partial pressures $\left(\mathrm{PCO}_{2}\right)$, oxygen partial pressures $\left(\mathrm{PO}_{2}\right)$, and oxygen saturations $\left(\mathrm{SO}_{2}\right)$ were assessed by a portable blood gas analyser (i-STAT, Abbott®, São Paulo, Brazil.). Fraction of inspired oxygen $\left(\mathrm{FiO}_{2}\right)$ was obtained directly by the anaesthetic machine (WATO EX-20, Shenzhen Mindray Bio-Medical Electronics Co., Ltd.).

A mathematical approach was done to determine the arterial oxygen content $\left(\mathrm{CaO}_{2}\right)$, central venous oxygen content $\left(\mathrm{CcvO}_{2}\right)$, capillary oxygen content $\left(\mathrm{CcO}_{2}\right)$ and pulmonary shunt fraction (V/Q), according to Lumb (2000) and Staub (1963). These parameters were calculated as follows:

$$
\begin{aligned}
& \mathrm{CaO}_{2}\left(\mathrm{~mL}^{\mathrm{dL}}{ }^{-1}\right)=(\mathrm{Hb} \times 1.34) \times\left(\mathrm{SaO}_{2}\right)+\left(\mathrm{PaO}_{2} \times 0.0031\right) ; \\
& \mathrm{CcvO}_{2}\left(\mathrm{~mL} \cdot \mathrm{dL}^{-1}\right)=(\mathrm{Hb} \times 1.34) \times\left(\mathrm{SvO}_{2}\right)+\left(\mathrm{PvO}_{2} \times 0.0031\right) ; \\
& \mathrm{CcO}_{2}\left(\mathrm{~mL}^{-1} \mathrm{dL}^{-1}\right)=(\mathrm{Hb} \times 1.34) \times\left(\mathrm{ScO}_{2}\right)+\left(\mathrm{PAO}_{2} \times 0.0031\right) ; \\
& \mathrm{V} / \mathrm{Q} \%=\left(\mathrm{CcO}_{2}-\mathrm{CaO}_{2}\right) /\left(\mathrm{CcO}_{2}-\mathrm{CcvO}_{2}\right) .
\end{aligned}
$$

Hemoglobin $(\mathrm{Hb})$ values were obtained by blood cell count $\left(\mathrm{g} . \mathrm{dL}^{-1}\right) ; 1.34$ is the oxygencarrying hemoglobin capacity $\left(\mathrm{mL} \cdot \mathrm{g}^{-1}\right) ; \mathrm{SaO}_{2}$ is the arterial oxygen saturation; $\mathrm{ScvO}_{2}$ is the central venous oxygen saturation; $\mathrm{ScO}_{2}$ is the pulmonary end-capillary oxygen saturation [assumed to be $100 \%$ (ie, 1)]; 0.0031 is the solubility coefficient of plasma oxygen; and $\mathrm{PAO}_{2}$ is the alveolar partial pressure of oxygen $(\mathrm{mmHg})$. 
The $\mathrm{PAO}_{2}$ was calculated as follows (LUMB, 2000): $\mathrm{PAO}_{2}=\left[\mathrm{FiO}_{2} *\left(\mathrm{~PB}-\mathrm{PH}_{2} \mathrm{O}\right)\right]-$ $\left(\mathrm{PaCO}_{2} / \mathrm{R}\right)$, where $\mathrm{FiO}_{2}$ is the fraction of inspired oxygen, $\mathrm{PB}$ is the local barometric pressure $(708,75 \mathrm{mmHg}) ; \mathrm{PH}_{2} \mathrm{O}$ is vapor pressure of water $(47 \mathrm{mmHg}), \mathrm{PaCO}_{2}$ is the arterial partial pressure of carbon dioxide, and $\mathrm{R}$ is the respiratory quotient, assumed to be 0.8 .

At the end of the treatment, anaesthesia was interrupted and animals were given 0.05 mg. $\mathrm{kg}^{-1}$ neostigmine IV and $0.1 \mathrm{mg} \cdot \mathrm{kg}^{-1}$ hyoscine IM for reversal of neuromuscular block. All sheep were assisted throughout the anaesthetic recovery and placed in sternal recumbency to eliminate ruminal gases by belching, and the tracheal tube was removed when the animals showed laryngotracheal reflex.

A G*Power Analysis test was used to determine the study sample size. Seven sheep were enough, with an actual power of $94 \%$. Data obtained were submitted to KolmogorovSmirnov normality test. All parametric variables were analysed by two-way ANOVA followed by Bonferroni's test for comparisons within each group, and by Tukey's test for moments among groups comparisons. Friedman's test was used for analysing the alveolar-arterial oxygen gradient $\left[\mathrm{P}(\mathrm{A}-\mathrm{a}) \mathrm{O}_{2}\right]$ and the arterial-end-tidal carbon dioxide gradient $\left[\mathrm{P}(\mathrm{a}-\mathrm{ET}) \mathrm{CO}_{2}\right]$. Paired sample t-test was used for $\mathrm{FiO}_{2}$, HR, MAP and FE'sevo comparisons between 60 and 120 min, within and between groups. Values were expressed as mean \pm standard deviation or as median and interquartile range, when appropriate. Significance level of $5 \%$ was considered $(p<0.05)$ accordingly.

\subsection{RESULTS}

Anaesthetic induction was considered satisfactory in all sheep, without complications. At 0 minute, arterial and central venous blood samples were collected after $229 \pm 58$ seconds for the dorsal recumbency group, $190 \pm 58$ seconds for the right lateral recumbency group, and $199 \pm 49$ seconds for the left lateral recumbency group, with no differences among them.

Differences were found on V/Q for the dorsal recumbency group, from 0 minute to 60 and $120 \mathrm{~min}(\mathrm{p}<0.001)$, which were same as that of the left lateral recumbency group $(\mathrm{p}=0.0012$ and $\mathrm{p}<0.001)$, respectively. For the right lateral recumbency group, difference was only found between 0 minute and $120 \mathrm{~min}(\mathrm{p}=0.0039)$. There was a difference observed in the groups only at $60 \mathrm{~min}$, when the V/Q was higher in the right lateral recumbency group as compared to the left one $(\mathrm{p}=0.0334)$ (Figure 1).

$\mathrm{CaO}_{2}, \mathrm{CcvO}_{2}, \mathrm{CcO}_{2}, \mathrm{PAO}_{2}, \mathrm{P}(\mathrm{A}-\mathrm{a}) \mathrm{O}_{2}$ values were significantly higher in all the groups, from 60 min after induction, and maintaining them further $(\mathrm{p}<0.05)\left(\right.$ Table 1). Higher $\mathrm{CaO}_{2}$ levels in animals from the left lateral recumbency group were observed at 0 min compared to 
the dorsal recumbency group $(\mathrm{p}=0.0177)$. No differences between treatments were observed for $\mathrm{CcvO}_{2}, \mathrm{CcO}_{2}$ and $\mathrm{PAO}_{2}$. The left recumbency group showed lower $\mathrm{P}(\mathrm{A}-\mathrm{a}) \mathrm{O}_{2}$ values at 0 $\min (p=0.0117)$ and $60 \mathrm{~min}$ compared to the right one $(\mathrm{p}=0.0282)$. No differences were found in $\mathrm{V}_{\mathrm{T}}$ and $\mathrm{V}_{\mathrm{M}}$ in all treatments and between time points, and the same was observed for $\mathrm{P}(\mathrm{a}-$ ET) $\mathrm{CO}_{2}$ values (Tables 1 and 2).

Main physiological variables are described in Table 2. $\mathrm{FiO}_{2}, \mathrm{HR}$ and FE'Sevo showed no statistical difference at different time points. Only the MAP was lower on the dorsal recumbency group at 120 min compared to the others $(\mathrm{p}=0.0425)$. Differences were observed in $\mathrm{pH}$ values at $60 \mathrm{~min}(\mathrm{p}=0.0173)$ and at $120 \mathrm{~min}(\mathrm{p}=0.0292)$ in the dorsal recumbency group, and at $60 \mathrm{~min}$ in the left lateral group when compared to 0 minute $(\mathrm{p}=0.0172)$. Furthermore, $\mathrm{pH}$ for the dorsal recumbency group was significantly higher when compared to the other treatments at $120 \mathrm{~min}(\mathrm{p}=0.0391) . \mathrm{PaO}_{2}$ showed higher values at 60 and $120 \mathrm{~min}$ for all the groups, when compared to the 0 minute ( $\mathrm{p}<0.001$ ). $\mathrm{PaCO}_{2}$ followed the same pattern, but only for the right and left recumbency groups. Conversely, the dorsal recumbency group showed stable $\mathrm{PaCO}_{2}$ throughout the anaesthesia period, thus, being different from the others at 120 $\min$.

\subsection{DISCUSSION}

Our study assessed the effect of three different recumbencies upon pulmonary shunt in sheep during a period of $120 \mathrm{~min}$ of sevoflurane anaesthesia. Collecting central venous blood samples is a simple, less expensive technique, and, in humans, less often associated with complications than withdrawing mixed venous blood by pulmonary artery catheters (WALLEY, 2011). Thus, measurement of $\mathrm{ScvO}_{2}$ has been validated as an option for $\mathrm{SvO}_{2}$ in healthy human patients (AKMAL et al., 2007). Central venous $\mathrm{Hb}$ saturated with oxygen has been measured from the right atrium or superior vena cava in human patients (WALTON \& HANSEN, 2018). However, some factors should be pointed out to make $\mathrm{ScvO}_{2}$ reliable, such as catheter placement, anatomy and the physiological state of the patient. Thus, if the patient has no clinical complications, it has been accepted that only a 2-3\% difference below is noted from mixed venous blood, which makes it a reliable index for all (REINHART, 2004; HARTOG \& BLOOS, 2014). Arterial and central venous oxygen saturations are essential variables used to calculate their respective oxygen content (COLLINS et al., 2015). The oxygen content indexes used in our study had been calculated based on blood gas analysis, which also included $\mathrm{SO}_{2}$ and $\mathrm{PO}_{2}$. Thus, we understand the reliability of our data lies precisely in the fact that all variables used to obtain the pulmonary shunt were calculated and not just estimated. 
The shunt was significantly higher in all groups right away after induction. Indeed, we expected low levels of $\mathrm{PaO}_{2}$ at 0 minute, at less than $60 \mathrm{mmHg}$, indicating that the animals were in severe hypoxaemia. This problem was due to the rumen distension and abdominal pressure at 0 minute, which led to severe $\mathrm{PaO}_{2}$ reduction following anaesthetic induction, even if $\mathrm{PaCO}_{2}$ levels were eucapnic (TRIM, 1981). $\mathrm{P}(\mathrm{A}-\mathrm{a}) \mathrm{O}_{2}$ values were lower as well, suggesting poor gas exchange efficiency for non-ventilated alveoli and anatomical shunt areas contributed to this condition (ROBINSON, 2009; ARAOS et al., 2012). Still, effects of ketamine induction might had a role over $\mathrm{PaO}_{2}$ levels at 0 minute. Although ketamine does not cause significant respiratory depression, pharyngeal and laryngeal reflexes remain uncoordinated and not protective, which might have caused bradypnea and even apnoea situations before intubation, and thus reducing $\mathrm{PaO}_{2}$ (SOLIMAN et al, 1975; STOELTING, 1999).

Despite hypoxaemia after induction, the shunt values had decreased from $60 \mathrm{~min}$ as a consequence of the mechanical ventilation and the high $\mathrm{FiO}_{2}$ as well. This is in accordance with studies conducted in horses (BRIGANTI et al., 2015) and sheep (ARAOS et al., 2012), that showed about $20-30 \%$ shunt at a $1.0 \mathrm{FiO}_{2}$. Likewise, the $\mathrm{PaO}_{2}$ values higher than $300 \mathrm{mmHg}$ are in accordance with those studies which had used similar $\mathrm{FiO}_{2}$, showing that both $\mathrm{FiO}_{2}$ and mechanical ventilation were effective on reversing hypoxaemia induction.

It was observed the $\mathrm{PaCO}_{2}$ increased in both lateral recumbencies, comparing to the 120 minute, but not for $\mathrm{ETCO}_{2}$ and $\mathrm{P}(\mathrm{a}-\mathrm{ET}) \mathrm{CO}_{2}$. Despite no statistical evidence for these two variables, at right lateral recumbency, there is clinical relevance showing an increasing tendency of $\mathrm{P}(\mathrm{a}-\mathrm{ET}) \mathrm{CO}_{2}$ overtime, which may reflect alveolar dead space (NUNN \& HILL, 1960), and some statistical difference may be found if treatments were kept for more than 120 min. In lateral recumbencies, the dependent lung (i.e., lowermost) is poorly ventilated due to atelectasis (PORCELLI, 1992). Thus, even high inspired oxygen concentrations would have minor effects over gas exchange and $\mathrm{CO}_{2}$ elimination (WEST, 1977). Even if shunt decreased over time, it was far from acceptable physiological values, i.e., less than 10\% (LUMB, 2000). Perhaps a PEEP maneuver could prevent alveolar collapse and promote alveolar recruitment, as observed in horses (HOPSTER et al., 2011).

It should be highlighted that $\mathrm{FiO}_{2}$ has a great influence over other variables. $\mathrm{FiO}_{2}$ at 0 minute (0.21) was far from the other moments (0.95) and it might have directly influenced the $\mathrm{PaO}_{2}$ and $\mathrm{P}(\mathrm{A}-\mathrm{a}) \mathrm{O}_{2}$ (OLIVEN et al., 1980), as pulmonary shunt ameliorated over time points. Thus, a statistical difference in the $\mathrm{P}(\mathrm{A}-\mathrm{a}) \mathrm{O}_{2}$ was expected among time points and even treatments. These values might be influenced by changes in $\mathrm{FiO}_{2}$, barometric pressure and body temperature (GILBERT \& KEIGHLEY, 1974). Blood flow from alveoli with low 
ventilation/perfusion ratio, non-ventilated alveoli, or anatomic shunt areas also contributed to elevated $\mathrm{P}(\mathrm{A}-\mathrm{a}) \mathrm{O}_{2}$ values (WEST, 1977), as observed for min 60 and $120 \mathrm{~min}$. In our study this may be due to the time that it took to equalise alveolar and pulmonary end-capillary $\mathrm{PO}_{2}$, which created a relative diffusion defect, or to $\mathrm{PO}_{2}$ losses, because of the very large partial pressure gradients, during 60 and $120 \mathrm{~min}$. This finding was in line with those found in anaesthetised sheep under different $\mathrm{FiO}_{2}$ values (ARAOS et al., 2012).

$\mathrm{V}_{\mathrm{M}}$ and $\mathrm{V}_{\mathrm{T}}$ values remained constant throughout the experimental period, with no statistical differences between time points and treatments, even with the presence of gravitational forces and visceral weight continuously pressing the diaphragm of these animals. Our study used pressure-controlled ventilation, in which an inspiratory flow was emitted by the ventilator by time (controlled cycle), and it determined a rapid increase in alveolar pressure. Maintaining constant pressure during the inspiratory phase allows alveoli to remain open and, theoretically, guarantees lung compliance and gas exchange (CORONA \& AUMANN, 2011), suggesting that this situation kept these variables constant. A similar situation was observed in cattle anaesthetised with sevoflurane under spontaneous ventilation (ARAÚJO et al., 2017), in which $\mathrm{V}_{\mathrm{M}}$ and $\mathrm{V}_{\mathrm{T}}$ remained without statistical difference throughout the 180-minute experimental period.

No statistical difference was observed in HR and MAP, except for MAP at $120 \mathrm{~min}$ from the sheep belonging to the dorsal recumbency group. This could be a result of impaired venous return in dorsal recumbency once gravitational forces impacted directly over rumen and other viscera, reducing venous blood flow (GENÇCELEP et al., 2004). The result in healthy patients is decreased stroke volume and pressure, because no changes are observed on vasomotor tone from heart beats (SINGH \& PINSKY, 2008).

Study limitations included: the absence of a $21 \% \mathrm{FiO}_{2}$ control group; the $2-5 \%$ below difference between central venous and mixed venous samples; and haemoglobin pattern changes during anaesthesia

\subsection{CONCLUSION}

This study demonstrates that all evaluated recumbencies did not interfere with pulmonary shunt. Clinically, the other results made shunt irrelevant, although high, since the oxygen tension contents and indexes were at physiological levels. 


\section{ACKNOWLEDGEMENTS}

The authors thank "São Paulo Research Foundation (FAPESP)" and "Coordination for the Improvement of Higher Education Personnel (CAPES)" for funding project $n^{\circ}$. 2018/15165-9.

\section{REFERENCES}

AKMAL, A. H. et al. The incidence of complications of central venous catheters at an intensive care unit. Annals of Thoracic Medicine. v. 2, p.61-63, 2007. Available from: <https://doi.org/10.4103/1817-1737.32232> Accessed dec. 20, 2020. doi: 10.4103/18171737.32232 .

ARAOS, J. D. et al. Use of the oxygen content-based index, Fshunt, as an indicator of pulmonary venous admixture at various inspired oxygen fractions in anesthetized sheep. American Journal of Veterinary Research. v. 73, p.2013-20, 2012. Available from: <https://doi.org/10.2460/ajvr.73.12.2013> Accessed dec. 20, 2020. doi: 10.2460/ajvr.73.12.2013.

ARAÚJO, M. A. et al. Cardiopulmonary effects of reverse Trendelenburg position at $5^{\circ}$ and $10^{\circ}$ in sevoflurane-anesthetized steers. Veterinary Anaesthesia and Analgesia. v. 44, p.854864, 2017. Available from: <https://doi.org/10.1016/j.vaa.2017.03.006> Accessed dec. 20, 2020. doi: 10.1016/j.vaa.2017.03.006.

BLAZE, C. A. et al. Effect of withholding feed on ventilation and the incidence of regurgitation during halothane anesthesia of adult cattle. American Journal of Veterinary Research. v. 49, p.2126-2129, 1988. Available from: <https://pubmed.ncbi.nlm.nih.gov/3149163/> Accessed dec. 20, 2020. PMID: 3149163

BRIGANTI, A. et al. Accuracy of different oxygenation indices in estimating intrapulmonary shunting at increasing infusion rates of dobutamine in horses under general anaesthesia. The Veterinary Journal. v. 204, p.351-356, 2015. Available from: <https://doi.org/10.1016/j.tvj1.2015.04.002> Accessed dec. 20, 2020. doi: 10.1016/j.tvj1.2015.04.002.

COLLINS, J. A. et al. Relating oxygen partial pressure, saturation and content: the haemoglobin - oxygen dissociation curve. Breathe. v. 11, p.194-201, 2015. Available from: $<$ https://breathe.ersjournals.com/content/11/3/194> Accessed jan. 17, 2021. doi: $10.1183 / 20734735.001415$.

CORONA, T. M.; AUMANN, M. Ventilator waveform interpretation in mechanically 
ventilated small animals. Journal of Veterinary Emergency - Critical Care. v. 21, p.496-14, 2011. Available from: <https://pubmed.ncbi.nlm.nih.gov/22316197/> Accessed jan. 17, 2021. doi: 10.1111/j.1476-4431.2011.00673.x.

DUECK, R. et al. Lung volume and VA/Q distribution response to intravenous versus inhalalation anesthesia in sheep. Anesthesiology. v. 61, p. 55-65, 1984. Available from: <https://pubmed.ncbi.nlm.nih.gov/6742484/> Accessed dec. 20, 2020. PMID: 6742484.

EGERMANN, M. et al. Animal models for fracture treatment in osteoporosis. Osteoporosis International. v. 16, p.129-38, 2005. Available from: <https://doi.org/10.1007/s00198-0051859-7> Accessed dec. 20, 2020. doi: 10.1007/s00198-005-1859-7.

EWING, K. K. Anesthesia techniques in sheep and goats. Veterinary Clinics of North America - Food Animal Practice. v. 6, p.759-778, 1990. Available from: $<$ https://doi.org/10.1016/S0749-0720(15)30845-8> Accessed dec. 20, 2020. doi: 10.1016/S0749-0720(15)30845-8.

FUJIMOTO. J. I.; LENEHAN, T. M. The influence of body position on the blood gas and acidbase status of halothane-anesthetized sheep. Veterinary Surgery. v. 14, p.169-172, 1985. Available from <https://doi.org/10.1111/j.1532-950X.1985.tb00855.X> Accessed dec. 20, 2020. doi: 10.1111/j.1532-950X.1985.tb00855.x.

FUJINO, Y. et al. Repetitive high-pressure recruitment maneuvers required to maximally recruit lung in a sheep model of acute respiratory distress syndrome. Critical Care Medicine. v. 29, p.1579-1586, 2001. Available from <https://doi.org/10.1097/00003246-20010800000014> Accessed dec. 20, 2020. doi: 10.1097/00003246-200108000-00014.

GALATOS, A. D. Anesthesia and analgesia in sheep and goats. Veterinary Clinics of North America - Food Animal Practice. v. 27, p.47-59, 2011. Available from <https://doi.org/10.1016/j.cvfa.2010.10.007> Accessed dec. 20, 2020. doi: 10.1016/j.cvfa.2010.10.007.

GENÇCELEP, M. et al. The effects of inhalation anaesthetics (halothane and isoflurane) on certain clinical and haematological parameters of sheep. Small Ruminant Research. v.53, p.157-60, 2004. Available from <https://doi.org/10.1016/j.smallrumres.2003.10.005> Accessed feb. 08, 2021. doi: 10.1016/j.smallrumres.2003.10.005.

GILBERT, R.; KEIGHLEY, J. The arterial-alveolar oxygen tension ratio. An index of gas exchange applicable to varying inspired oxygen concentrations. American Review of 
Respiratory Diseases. v. 18, p.1043-1048, 1974. Available from <https://pubmed.ncbi.nlm.nih.gov/4809154/> Accessed feb. 08, 2021. doi: 10.1164/arrd.1974.109.1.142.

HARTOG, C.; BLOOS, F. Venous oxygen saturation. Best Practice \& Research Clinical Anaesthesiology. $\quad$ v. 28, p.419-428, 2014. Available from <https://doi.org/10.1016/j.bpa.2014.09.006> Accessed jan. 16, 2021. doi: 10.1016/j.bpa.2014.09.006.

HOPSTER, K. et al. Intermittent positive pressure ventilation with constant positive endexpiratory pressure and alveolar recruitment manoeuvre during inhalation anaesthesia in horses undergoing surgery for colic, and its influence on the early recovery period. Veterinary Anaesthesia and Analgesia. v. 38, p.169-177, 2011. Available from <https://pubmed.ncbi.nlm.nih.gov/21492381/> Accessed feb. 08, 2021. doi: 10.1111/j.14672995.2011.00606.x.

LOVERING, A. T. et al. Transpulmonary shunting into the general circulation: An update. Injury. v. 41, p.1-20, 2015. Available from <https://doi.org/10.1016/S0020-1383(10)70004-8> Accessed feb. 10, 2021. doi: 10.1016/S0020-1383(10)70004-8.

LUMB, A. B. Nunn's Applied Respiratory Physiology. 5th. ed. Oxford: ButterworthHeinemann; 2000.

MCMILLEN, C. The sheep - an ideal model for biomedical research? Anzccart News. v. 14, p.1-4, 2001. Available from <https://anzccart.adelaide.edu.au/system/files/media/documents/2019-07/news0601.pdf> Accessed nov. 11, 2020.

MEYER, H. et al. Cardiopulmonary effects of dorsal recumbency and high-volume caudal epidural anaesthesia with lidocaine or xylazine in calves. The Veterinary Journal. v. 186, p.316-22, 2010. Available from <https://doi.org/10.1016/j.tvj1.2009.08.020> Accessed dec. 20, 2020. doi: 10.1016/j.tvj1.2009.08.020.

NUNN, J. F.; HILL, D. W. Respiratory dead space and arterial to end-tidal $\mathrm{CO}_{2}$ tension difference in anesthetized man. Journal of Applied Physiology. v. 15, p.383-389, 1960. Available from <https://pubmed.ncbi.nlm.nih.gov/14427915/> Accessed dec. 20, 2020. doi: 10.1152/jappl.1960.15.3.383.

OLIVEN, A. et al. Influence of varying inspired oxygen tensions on the pulmonary venous 
admixture (shunt) of mechanically ventilated patients. Critical Care Medicine. v. 8, p.99-101, 1980. Available from <https://pubmed.ncbi.nlm.nih.gov/6986232/> Accessed feb. 15, 2021. doi: 10.1097/00003246-198002000-00009.

PETERSSON, J.; GLENNY, R. W. Gas exchange and ventilation-perfusion relationships in the lung. European Respiratory Journal. v. 44, p.1023-1041, 2014. Available from $<$ https://doi.org/10.1183/09031936.00037014> Accessed dec. 20, 2020. doi: 10.1183/09031936.00037014.

PORCELLI, R. J. Pulmonary hemodynamics. Treatise on Pulmonary Toxicology. Vol. 1: Comparative Biology of the Normal Lung. v. 52, p.241-70, 1992. Available from <https://www.ncbi.nlm.nih.gov/pmc/articles/PMC1128368/> Accessed feb. 20, 2021. PMCID: PMC1128368.

REINHART, K. Continuous central venous and pulmonary artery oxygen saturation monitoring in the critically ill. Critical Care Medicine. v. 52, p.1572-1578, 2004. Available from <https://doi.org/10.1007/s00134-004-2337-y> Accessed dec. 20, 2020. doi: 10.1007/s00134004-2337-y.

ROBINSON, N. E. The respiratory system. In: MUIR, W. W.; HUBBELL, J. A. E., editors. Equine Anesthesia: Monitoring and Emergency Therapy. 2nd. ed. St Louis, MO: Saunders Elsevier; 2009.

SINGH, I.; PINSKY, M. R. Heart-Lung Interactions. 1st. ed. Elsevier Inc, 2008.

STAUB, N. C. Alveolar-arterial oxygen tension gradient due to diffusion. Journal of Applied Physiology. v. 18, p.673-80, 1963. Available from $<$ https://pubmed.ncbi.nlm.nih.gov/13983535/> Accessed dec. 20, 2020. doi: 10.1152/jappl.1963.18.4.673.

TRANQUILLI, W. J. Techniques of inhalation anesthesia in ruminants and swine. Veterinary Clinics of North America - Food Animal Practice. v. 2, p.593-619, 1986. Available from <https://doi.org/10.1016/S0749-0720(15)31208-1> Accessed dec. 20, 2020. doi: 10.1016/S0749-0720(15)31208-1.

TRIM, C. M. Sedation and general anesthesia in ruminants. California Veterinary. v. 35, p.29$36,1981$.

WALLEY, K. R. Use of central venous oxygen saturation to guide therapy. Concise Clinical Review. v. 184, p.514-520, 2011. Available from <https://doi.org/10.1164/rccm.201010- 
1584CI> Accessed feb. 25, 2021. doi: 10.1164/rccm.201010-1584CI.

WALTON, R. A. L.; HANSEN, B. D. Venous oxygen saturation in critical illness. v. 28, p.387397, 2018. Available from <https://doi.org/10.1111/vec.12749> Accessed jan. 6, 2021. doi: 10.1111/vec.12749.

WEST, J. B. Ventilation-perfusion relationships. American Review of Respiratory Diseases. v. 116, p.919-943, 1977. Available from <https://www.atsjournals.org/doi/abs/10.1164/arrd.1977.116.5.919?journalCode=arrd> Accessed dec. 20, 2020. doi: 10.1164/arrd.1977.116.5.919. 

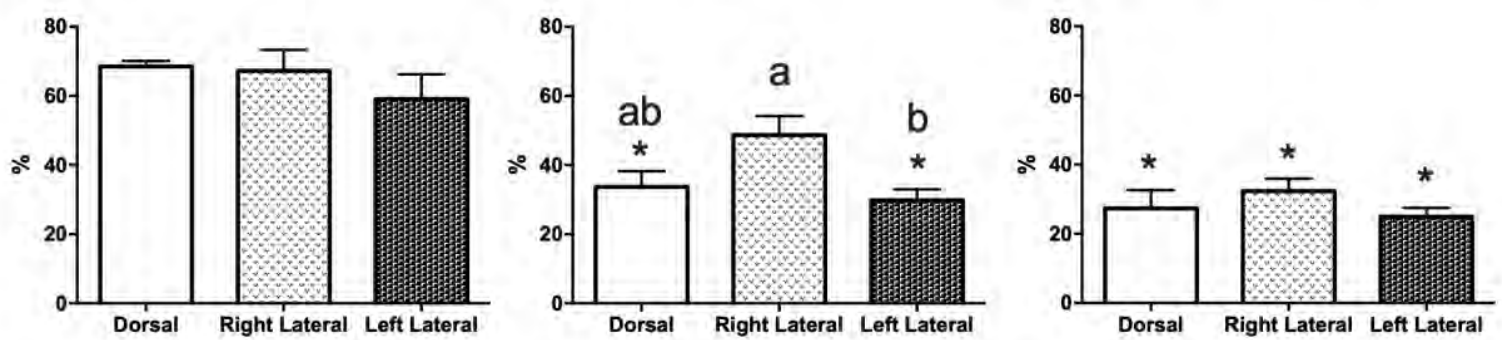

Figure $1-$ Mean \pm ED of the pulmonary shunt fraction (V/Q) of sevoflurane-anaesthetized sheep, mechanically-ventilated under dorsal, right lateral or left lateral recumbencies. * Statistical difference from 0 minute; Different letters indicate statistical difference between groups $(\mathrm{P} \leq 0.05)$. 
Table 1 - Oxygen tension, content indexes and parameters of sevoflurane-anaesthetised sheep, mechanically-ventilated under dorsal, right lateral or left lateral recumbencies.

\begin{tabular}{|c|c|c|c|c|}
\hline Parameter & Treatment & 0 minute & $60 \mathrm{~min}$ & $120 \mathrm{~min}$ \\
\hline \multirow{3}{*}{$\underset{\left(\mathbf{m L} \cdot \mathbf{d L}^{-1}\right)}{\mathbf{C a O}_{2}}$} & Dorsal & $8.9 \pm 1.4^{\mathrm{a}}$ & $23.0 \pm 3.8^{*}$ & $23.7 \pm 3.8^{*}$ \\
\hline & Right Lateral & $9.7 \pm 1.6^{\mathrm{ab}}$ & $20.8 \pm 3.4^{*}$ & $23.5 \pm 2.6^{*}$ \\
\hline & Left Lateral & $9.9 \pm 1.4^{\mathrm{b}}$ & $23.6 \pm 1.7^{*}$ & $23.9 \pm 2.7 *$ \\
\hline \multirow{3}{*}{$\underset{\left(\mathbf{m L}^{-d_{2}}{ }^{-1}\right)}{\mathrm{CcvO}_{2}}$} & Dorsal & $7.0 \pm 1.2$ & $12.3 \pm 3.0^{*}$ & $11.9 \pm 2.9 *$ \\
\hline & Right Lateral & $7.7 \pm 1.4$ & $12.8 \pm 3.6^{*}$ & $13.6 \pm 3.7^{*}$ \\
\hline & Left Lateral & $7.7 \pm 2.2$ & $12.7 \pm 3.7 *$ & $12.2 \pm 2.9^{*}$ \\
\hline \multirow{3}{*}{$\underset{\left(\mathbf{m L} . \mathbf{d L}^{-1}\right)}{\mathbf{C c O}_{2}}$} & Dorsal & $13.5 \pm 2.9$ & $28.3 \pm 2.4^{*}$ & $28.2 \pm 2.4 *$ \\
\hline & Right Lateral & $13.6 \pm 2.4$ & $28.4 \pm 2.0^{*}$ & $28.1 \pm 2.1 *$ \\
\hline & Left Lateral & $13.2 \pm 2.6$ & $28.2 \pm 2.3^{*}$ & $27.9 \pm 2.4^{*}$ \\
\hline \multirow{3}{*}{$\underset{(\mathbf{m m H g})}{\mathrm{PaO}_{2}}$} & Dorsal & $47.2 \pm 4.4$ & $408.8 \pm 53.3^{*}$ & $433.0 \pm 61.6^{*}$ \\
\hline & Right Lateral & $48.6 \pm 9.7$ & $333.0 \pm 80.1 *$ & $417.8 \pm 42.1 *$ \\
\hline & Left Lateral & $51.4 \pm 6.0$ & $429.8 \pm 43.0^{*}$ & $438.8 \pm 41.2^{*}$ \\
\hline \multirow{3}{*}{$\begin{array}{c}\mathrm{PAO}_{2} \\
(\mathbf{m m H g})\end{array}$} & Dorsal & $102.1 \pm 18.5$ & $579.9 \pm 9.4^{*}$ & $576.5 \pm 7.7^{*}$ \\
\hline & Right Lateral & $100.9 \pm 8.8$ & $577.2 \pm 11.8^{*}$ & $567.7 \pm 8.2^{*}$ \\
\hline & Left Lateral & $94.3 \pm 3.8$ & $576.2 \pm 13.1^{*}$ & $567.6 \pm 9.2 *$ \\
\hline \multirow{3}{*}{$\begin{array}{c}\mathrm{P}(\mathrm{A}-\mathbf{a}) \mathrm{O}_{2} \\
(\mathrm{mmHg})\end{array}$} & Dorsal & $45(43 ; 71)^{\mathrm{ab}}$ & $162(133 ; 212)^{\mathrm{ab} *}$ & $117(93 ; 218)^{*}$ \\
\hline & Right Lateral & $49(43 ; 72)^{\mathrm{a}}$ & $225(184 ; 294)^{a *}$ & $139(110 ; 197)^{*}$ \\
\hline & Left Lateral & $39(37 ; 52)^{b}$ & $145(119 ; 173)^{\mathrm{b} *}$ & $126(81 ; 176)^{*}$ \\
\hline \multirow{3}{*}{$\begin{array}{c}\mathrm{PaCO}_{2} \\
(\mathrm{mmHg})\end{array}$} & Dorsal & $32.8 \pm 7.2$ & $39.0 \pm 8.8$ & $39.9 \pm 8.4^{b}$ \\
\hline & Right Lateral & $30.4 \pm 7.1$ & $44.6 \pm 11.8^{*}$ & $50.5 \pm 12.0^{* a}$ \\
\hline & Left Lateral & $35.8 \pm 3.8$ & $45.5 \pm 7.2^{*}$ & $50.6 \pm 7.7 * a$ \\
\hline \multirow{3}{*}{$\mathrm{PaO}_{2} / \mathrm{FiO}_{2}$} & Dorsal & 223 & 429 & 455 \\
\hline & Right Lateral & 228 & 346 & 438 \\
\hline & Left Lateral & 242 & 446 & 461 \\
\hline \multirow{3}{*}{$\begin{array}{c}\mathrm{P}(\mathbf{a}-\mathrm{ET}) \mathrm{CO}_{2} \\
(\mathrm{mmHg})\end{array}$} & Dorsal & - & $6.8(4.2 ; 8.5)$ & $5.8(4.5 ; 10.0)$ \\
\hline & Right Lateral & - & $6.6(2.6 ; 12.0)$ & $8.8(6.0 ; 9.4)$ \\
\hline & Left Lateral & - & $9.4(4.9 ; 10.0)$ & $8.3(2.1 ; 9.7)$ \\
\hline
\end{tabular}


$\mathrm{CaO}_{2}$ : arterial oxygen content. $\mathrm{CcvO}_{2}$ : central venous oxygen content. $\mathrm{CcO}_{2}$ : pulmonary endcapillary oxygen content. $\mathrm{PaO}_{2}$ : arterial partial pressure of oxygen. $\mathrm{PAO}_{2}$ : alveolar oxygen partial pressure. $\mathrm{P}(\mathrm{A}-\mathrm{a}) \mathrm{O}_{2}$ : alveolar-arterial oxygen gradient. $\mathrm{PaCO}_{2}$ : arterial partial pressure of carbon dioxide. $\mathrm{PaO}_{2} / \mathrm{FiO}_{2}$ : arterial partial pressure of oxygen and fraction of inspired oxygen ratio. $\mathrm{P}(\mathrm{a}-\mathrm{ET}) \mathrm{CO}_{2}$ : arterial-end-tidal carbon dioxide gradient. * Statistical difference from 0 minute; Different letters indicate statistical difference between groups $(\mathrm{P} \leq 0.05)$. Values expressed by mean $\pm \mathrm{SD}$. $\mathrm{P}(\mathrm{A}-\mathrm{a}) \mathrm{O}_{2}$ and $\mathrm{P}(\mathrm{a}-\mathrm{ET}) \mathrm{CO}_{2}$ expressed as median and interquartile range. 
Table 2 - Physiological and blood gas parameters of sevoflurane-anaesthetised sheep, mechanically-ventilated under dorsal, right lateral or left lateral recumbencies.

\begin{tabular}{|c|c|c|c|c|}
\hline Parameter & Treatment & 0 minute & $60 \mathrm{~min}$ & $120 \mathrm{~min}$ \\
\hline \multirow{3}{*}{$\begin{array}{c}\mathrm{FiO}_{2} \\
(\%)\end{array}$} & Dorsal & 0.21 & $0.95 \pm 0.01$ & $0.95 \pm 0.02$ \\
\hline & Right Lateral & 0.21 & $0.96 \pm 0.02$ & $0.95 \pm 0.03$ \\
\hline & Left Lateral & 0.21 & $0.96 \pm 0.02$ & $0.95 \pm 0.01$ \\
\hline \multirow{3}{*}{$\begin{array}{c}\mathrm{PcvO}_{2} \\
(\mathrm{mmHg})\end{array}$} & Dorsal & $33.6 \pm 3.6$ & $86.8 \pm 37.7 *$ & $73.3 \pm 21.9^{*}$ \\
\hline & Right Lateral & $36.5 \pm 4.9$ & $95.5 \pm 54.3^{*}$ & $117.8 \pm 67.4 *$ \\
\hline & Left Lateral & $36.1 \pm 3.6$ & $98.3 \pm 58.1 *$ & $104.1 \pm 60.1 *$ \\
\hline \multirow{3}{*}{$\begin{array}{l}\mathrm{SaO}_{2} \\
(\%)\end{array}$} & Dorsal & $0.73 \pm 0.05 b$ & $1.0^{*}$ & $1.0^{*}$ \\
\hline & Right Lateral & $0.77 \pm 0.06^{\mathrm{ab}}$ & $1.0^{*}$ & $1.0^{*}$ \\
\hline & Left Lateral & $0.81 \pm 0.05^{\mathrm{a}}$ & $1.0^{*}$ & $1.0^{*}$ \\
\hline \multirow{3}{*}{$\begin{array}{c}\mathrm{ScvO}_{2} \\
(\%)\end{array}$} & Dorsal & $0.58 \pm 0.08$ & $0.93 \pm 0.07 *$ & $0.93 \pm 0.05 *$ \\
\hline & Right Lateral & $0.62 \pm 0.07$ & $0.93 \pm 0.05^{*}$ & $0.94 \pm 0.06^{*}$ \\
\hline & Left Lateral & $0.61 \pm 0.08$ & $0.94 \pm 0.05^{*}$ & $0.92 \pm 0.08 *$ \\
\hline \multirow{3}{*}{$\mathbf{T}^{\circ} \mathbf{C}$} & Dorsal & $38.3 \pm 0.4$ & $37.3 \pm 0.5$ & $37.0 \pm 0.8$ \\
\hline & Right Lateral & $38.5 \pm 0.5$ & $37.6 \pm 1.1$ & $37.2 \pm 0.8$ \\
\hline & Left Lateral & $38.2 \pm 0.4$ & $37.2 \pm 0.8$ & $36.7 \pm 0.8$ \\
\hline \multirow{3}{*}{$\begin{array}{l}\mathrm{ETCO}_{2} \\
\mathrm{mmHg}\end{array}$} & Dorsal & - & $32.5 \pm 7.7$ & $33.0 \pm 10.9^{\mathrm{b}}$ \\
\hline & Right Lateral & - & $38.0 \pm 6.9$ & $42.5 \pm 10.4^{\mathrm{a}}$ \\
\hline & Left Lateral & - & $37.5 \pm 5.5$ & $41.6 \pm 6.8^{\mathrm{a}}$ \\
\hline \multirow{3}{*}{$\begin{array}{c}\text { ET'Sevo } \\
(\%)\end{array}$} & Dorsal & - & $1.80 \pm 0.14$ & $1.83 \pm 0.22$ \\
\hline & Right Lateral & - & $1.73 \pm 0.18$ & $2.00 \pm 0.28$ \\
\hline & Left Lateral & - & $1.82 \pm 0.15$ & $2.00 \pm 0.21$ \\
\hline
\end{tabular}

$\mathrm{FiO}_{2}$ : fraction of inspired oxygen. $\mathrm{PcvO}_{2}$ : central venous partial pressure of oxygen. $\mathrm{SaO}_{2}$ : arterial oxygen saturation. $\mathrm{ScvO}_{2}$ : central venous oxygen saturation. $\mathrm{T}^{\circ} \mathrm{C}$ : body temperature. $\mathrm{ETCO}_{2}$ : end-tidal carbon dioxide. ET'Sevo: end-tidal sevoflurane concentration. Values expressed by mean $\pm \mathrm{SD}$. $*$ Statistical difference from 0 minute; Different letters indicate statistical difference between groups $(\mathrm{P} \leq 0.05)$. 


\section{APÊNDICE A}

Roberto Romano do Prado Filho <roberto.romano.filho@usp.br>

Ciência Rural - Manuscript ID CR-2021-0251

1 message

Ciência Rural <onbehalfof@manuscriptcentral.com>

Reply-To: cienciarural@gmail.com

To: carregaro@usp.br

Cc: roberto.romano.filho@usp.br, marianacsanchess@usp.br, carregaro@usp.br

29-Mar-2021

Dear Dr. Carregaro:

Your manuscript entitled "Influence of recumbency on the pulmonary shunt in sevoflurane-anaesthetised sheep" has been successfully submitted online and is presently being given full consideration for publication in the

Ciência Rural.

Your manuscript ID is CR-2021-0251.

Please mention the above manuscript ID in all future correspondence or when calling the office for questions. If there are any changes in your street address or e-mail address, please log in to ScholarOne Manuscripts at https://mc04.manuscriptcentral.com/cr-scielo and edit your user information as appropriate.

You can also view the status of your manuscript at any time by checking your Author Center after logging in to https://mc04.manuscriptcentral.com/cr-scielo.

Thank you for submitting your manuscript to the Ciência Rural.

Sincerely,

Ciência Rural Editorial Office 